\title{
Weighting by iteration: the case of Ryll-Nardzewski's iterations
}

\author{
Lucio R. Berrone*
}

\begin{abstract}
Aczél's and Ryll-Nardzewski's dyadic iterations are iterative procedures which associate to a given mean $M$ a family of means $\left\{M^{d}: d \in\right.$ Dyad $([0,1])\}$ parameterized by Dyad $([0,1])$, the dyadic fractions of the interval $[0,1]$. Aczél's iterations exhibit a nice characteristic: when $M$ is a strict continuous mean and $x<y$, the set $\left\{M^{d}(x, y): d \in \operatorname{Dyad}([0,1])\right\}$ is dense in $[x, y]$. This fact is in the basis of the construction of an algorithm of weighting for an ample class of means. In pursuit of a similar algorithm using Ryll-Nardzewski's instead of Aczél's iterations, a series of obstacles is found, which motivates the detailed study of these last conducted along this paper. Among other result of interest, several conditions on the mean $M$ are identified which make viable a weighting algorithm based on these iterations.
\end{abstract}

AMS Mathematical Subject Classification (2010): 26E60, 39B12, $26 \mathrm{~A} 15$.

\section{Introduction}

Let be given a real interval $I$ and a function $F: I \times I \rightarrow I$. Denoting by $\operatorname{Dyad}([0,1])$ the set of dyadic fractions of the interval $[0,1]$; i.e.

$$
\operatorname{Dyad}([0,1])=\left\{\frac{k}{2^{n}}: 0 \leq k \leq 2^{n}, n \in \mathbb{N}_{0}\right\},
$$

let us define $F^{(d)}$ and $F^{[d]}$ for $d \in \operatorname{Dyad}([0,1])$ as certain sequences of compositions of $F$ with itself. In the first place, the Aczél's dyadic iterations $F^{(d)}$ of $F$ are inductively defined for $x, y \in I$ as follows:

$$
F^{(0)}(x, y) \equiv x, F^{(1)}(x, y) \equiv y,
$$

and, for every $n \in \mathbb{N}_{0}$ and $0 \leq k \leq 2^{n+1}$,

$$
F^{\left(\frac{k}{2^{n+1}}\right)}(x, y)=\left\{\begin{array}{ll}
F^{\left(\frac{h}{2^{n}}\right)}(x, y), & k=2 h, 0 \leq h \leq 2^{n} \\
F\left(F^{\left(\frac{h}{2^{n}}\right)}(x, y), F^{\left(\frac{h+1}{2^{n}}\right)}(x, y)\right), & k=2 h+1,0 \leq h \leq 2^{n}-1
\end{array} .\right.
$$

${ }^{*}$ Consejo Nacional de Investigaciones Científicas y Técnicas (CONICET), Laboratorio de Acústica y Electroacústica, Facultad de Cs. Exactas, Ing. y Agrim., Univ. Nac. de Rosario, Riobamba 245 bis, 2000-Rosario, Argentina; e-mail address: berrone@fceia.unr.edu.ar 
In the second place, the Ryll-Nardzewski's dyadic iterations $F^{[d]}$ of $F$ are defined for $x, y \in I$ by

$$
F^{[0]}(x, y) \equiv x, F^{[1]}(x, y) \equiv y,
$$

and, for every $n \in \mathbb{N}_{0}$ and $0 \leq k \leq 2^{n+1}$,

$$
F^{\left[\frac{k}{2^{n+1}}\right]}(x, y)=\left\{\begin{array}{ll}
F\left(x, F^{\left[\frac{k}{2^{n}}\right]}(x, y)\right), & 0 \leq k \leq 2^{n} \\
F\left(F^{\left[\frac{k-2^{n}}{2^{n}}\right]}(x, y), y\right), & 2^{n} \leq k \leq 2^{n+1}
\end{array} .\right.
$$

Remarkably, both dyadic iterations arose in mathematical constructions related to a characterization of the quasiarithmetic means by using functional equations. Indeed, Aczél's dyadic iterations were employed in [1] (see also [2], Chap. 6 and [3], Chap. 17) to characterize quasiarithmetic means as the solutions to

$$
F(F(x, y), F(u, v))=F(F(x, u), F(y, v)), x, y, u, v \in I,
$$

a functional equation of composite type named bisymmetry equation, while RyllNardzewski's ones arose in [22] with the same purpose but using, instead of (6), the system of equations

$$
\left\{\begin{array}{l}
F(F(x, y), z)=F(F(x, z), F(y, z)) \\
F(x, F(y, z))=F(F(x, y), F(x, z))
\end{array} \quad x, y, z \in I\right.
$$

known as self-distributivity equations.

In this paper the above defined iterations are studied mainly in the case in which the function $F$ is a mean, so that a relative series of notations and terminology is now presented. Remind that a (two variables) mean $M$ defined on a real interval $I$ is a function $M: I \times I \rightarrow I$ which is internal; i.e., it satisfies the property

$$
\min \{x, y\} \leq M(x, y) \leq \max \{x, y\}, x, y \in I .
$$

The mean is said to be strict when the inequalities in (8) are strict provided that $x \neq y$ (strict internality). In view of the equality

$$
M(x, x)=x, x \in I,
$$

holds for every internal function $M$, means are reflexive functions. A mean $M$ is said to be symmetric when

$$
M(x, y)=M(y, x), x, y \in I .
$$

The coordinate means $X(x, y) \equiv x$ and $Y(x, y) \equiv y$ are the unique means depending on a sole variable. The functions at the leftmost and rightmost members of the inequalities (8) are the extremal means min and max.

A mean $M$ is [strictly] isotone when $x \mapsto M(x, y)$ is [strictly] increasing for every $y \in I$ and $y \mapsto M(x, y)$ is [strictly] increasing for every $x \in I$. An isotone mean preserve the product order in $I^{2}$; i.e., $M(x, y) \leq M\left(x^{\prime}, y^{\prime}\right)$ provided that $(x, y) \leq\left(x^{\prime}, y^{\prime}\right)$. A strictly isotone mean is a strict mean. 
If $M$ is a mean defined on $I$ and $f: I \rightarrow \mathbb{R}$ is a strictly monotonic and continuous function (i.e., a homeomorphism from $I$ onto $f(I)$ ), the $f$-conjugated $M_{f}$ of $M$ is the mean defined on $f(I)$ by

$$
M_{f}(x, y)=f\left(M\left(f^{-1}(x), f^{-1}(y)\right), x, y \in f(I) .\right.
$$

When $M$ is a given mean and $f$ varies on the set of homeomorphism from $I$ in $\mathbb{R}$, then $M_{f}$ runs along the entire class of conjugation of $M$. The means which are conjugated of the arithmetic mean $A(x, y)=(x+y) / 2$ are named quasiarithmetic means; thus, a quasiarithmetic mean has the form

$$
A_{f}(x, y)=f\left(\frac{f^{-1}(x)+f^{-1}(y)}{2}\right), x, y \in I,
$$

where $f: I \rightarrow \mathbb{R}$ is a strictly monotonic and continuous function.

When the interval $I$ is a cone, a mean $M$ defined on $I$ is said to be homogeneous provided that $M_{f}=M$ for every linear map $f: I \rightarrow I, f \neq 0$.

A mean $M$ is said to be continuous or separately continuous when $(x, y) \mapsto$ $M(x, y)$ is continuous on $I^{2}$ or $x \mapsto M(x, y), y \in I$, and $y \mapsto M(x, y), x \in I$, are continuous on $I$, respectively. Means fulfilling special types of separate uniform Lipschitz-continuity will play a capital role in this paper. If, for every $x, y \in I$, the inequalities

$$
\left|M(x, u)-M\left(x, u^{\prime}\right)\right| \leq\left|u-u^{\prime}\right| \text { and }\left|M(u, y)-M\left(u^{\prime}, y\right)\right| \leq\left|u-u^{\prime}\right|
$$

hold for every $u, u^{\prime} \in I$, then the mean $M$ is said to be separately nonexpansive, while it is said separately quasicontractive when it is separately nonexpansive and, for every $x, y \in I$, one inequality (12) at least is strict provided that $u, u^{\prime} \in I, u \neq u^{\prime}$. In view of the inequalities

$$
\begin{aligned}
\left|M(x, y)-M\left(x^{\prime}, y^{\prime}\right)\right| & \leq\left|M(x, y)-M\left(x^{\prime}, y\right)\right|+\left|M\left(x^{\prime}, y\right)-M\left(x^{\prime}, y^{\prime}\right)\right| \\
& \leq\left|x-x^{\prime}\right|+\left|y-y^{\prime}\right|, x, y, x^{\prime}, y^{\prime} \in I,
\end{aligned}
$$

every separately nonexpansive mean $M$ turns out to be a $\left(L^{1}\right)$ Lipschitz-continuous function (with Lipschitz constant equal to 1). Extremal and coordinate means are simple examples of separately nonexpansive and separately quasicontractive means, respectively.

Separate nonexpansiveness and quasicontractiveness are properties of a mean which are not generally preserved by conjugacy (see Example 28 in the Appendix). Unlike these properties, the following ones remain invariant under conjugacy: a mean $M$ is said to be separately $(C)$-nonexpansive [separately (C)-quasicontractive] when the class of conjugation of $M$ contains a separately nonexpansive [separately quasicontractive] mean; i.e., when there exists a homeomorphism $f: I \rightarrow \mathbb{R}$ such that $M_{f}$ is separately nonexpansive [separately quasicontractive] (on $f(I)$ ). Further discussion on these concepts is contained in the final Appendix.

Returning to the main exposition, it should be pointed out that, under the denomination of dyadic iterations, a particularized study of Aczél's dyadic iterations was conducted in [8] and [4] (see also [11]). In the last of this references, the following result was proved: 
Theorem 1 For a strictly internal and reflexive function $M$, the function $d \mapsto$ $M^{(d)}(x, y)$ defined on $\operatorname{Dyad}([0,1])$ is monotonically extended to $\delta \in[0,1]$ by

$$
M^{(\delta)}(x, y)=\lim _{d_{n} \uparrow \delta} M^{\left(d_{n}\right)}(x, y), x, y \in I,
$$

where $\left(d_{n}: n \in \mathbb{N}\right) \subseteq \operatorname{Dyad}([0,1])$ is an increasing sequence with $\lim _{n \uparrow+\infty} d_{n}=$ $\delta$. The extension $\delta \mapsto M^{(\delta)}(x, y)$, which is a strictly monotonic function when $x \neq y$ (strictly increasing or decreasing depending on $x<y$ or $x>y$ ), turns out to be unique and continuous provided that $M$ is continuous. In this case, $(x, y) \mapsto M^{(\delta)}(x, y)$ is continuous in $I^{2}$ for every $\delta \in[0,1]$.

Now, when $M$ is a strict continuous mean, the map $\delta \mapsto M^{(\delta)}(x, y)$ in the statement of Theor. 1 exhibits a close similitude with the map

$$
\delta \mapsto(1-\delta) x+\delta y
$$

In fact, besides of being strictly monotonic and continuous, both maps assume the values $x$ and $y$ at the extreme points $\delta=0$ and $\delta=1$, respectively. Furthermore, $M^{(1 / 2)}(x, y)=M(x, y)$, while the map (14) takes the value $(x+y) / 2=A(x, y)$ at $\delta=1 / 2$. As it is well known, (14) is the weighted version of the arithmetic mean $A(x, y)$, so that the map $\delta \mapsto M^{(\delta)}$ can be thought as a sort of weighting of the mean $M$. In conformity with this thought, it can be inductively proved that, for every $d \in \operatorname{Dyad}([0,1])$,

$$
A^{(d)}(x, y)=(1-d) x+d y,
$$

and hence, that the continuous extension $A^{(\delta)}$ of $A^{(d)}$, whose existence and uniqueness is ensured by Theor. 1 , is given by (the weighting) $A^{(\delta)}(x, y)=$ $(1-\delta) x+\delta y$ of $A$.

The problem of constructing weighted versions of a given mean is an important problem in the Theory of Means. General references on this theme are [14] and [13], while [18], [19], [20] and [21] is list of articles in which the problem of weighting is solved for particular means. Even though this is a very incomplete list, the state of the art is revealed by its items: the weighting of a mean $M$ (or, sometimes, a mean belonging to certain family $\mathcal{M}$ of means) is obtained by suitably modifying an analytical representation of $M$ (or of the mean in $\mathcal{M}$ ). A theory of weightings of two variables means which is independent of particular representations has arisen in [5], while the case of $n$ variables means was treated in [7]. An extensive discussion of the problem of weighting of means is also contained in this last reference. A generalization of the Aczél's dyadic iterations to three variables means is studied in [12].

Following [5], given a (two variables) mean $M$ defined on $I$, by a weighting $M^{\delta}$ of $M$ is understood a map $\delta \mapsto M^{\delta}$ defined on the unit interval $[0,1]$ such that

(CW1) $M^{\delta}$ is a mean for every $\delta \in[0,1]$; and 
(CW2) for every $x, y \in I, \delta \mapsto M^{\delta}(x, y)$ assumes the particular values $M^{0}(x, y)=$ $x, M^{1 / 2}(x, y)=M(x, y)$ and $M^{1}(x, y)=y$ at the points $\delta=0,1 / 2,1$, respectively.

The problem of finding a weighting of a general (continuous) mean $M$ becomes non trivial when continuity or monotonicity of the map $\delta \mapsto M^{\delta}$ is required. In this regard, Theor. 1 is a satisfactory result inasmuch as it provides, for the means belonging to a class as ample as that constituted by the continuous strict means, a continuous and strictly monotonic weighting.

In this paper, the question of determining the conditions under which the Ryll-Nardzewski's iterations of a mean $M$ can be profited to construct a weighting of $M$ is investigated. In this regard, it should be noted that the first iterations of Ryll-Nardzewski's coincide with the Aczél's first ones: $M^{\left[k / 2^{n}\right]}=$ $M^{\left(k / 2^{n}\right)}$ if $n \leq 2$ and $0 \leq k \leq 2^{n}$. Moreover, both dyadic iterations coincide on the class of quasiarithmetic means; namely, if $M$ has the form (11), then

$$
M^{[d]}(x, y)=f\left(\frac{(1-d) f^{-1}(x)+d f^{-1}(y)}{2}\right)=M^{(d)}(x, y), x, y \in I .
$$

On the other hand, it is easy to see that an alternative definition of Aczél's dyadic iterations can be given by $M^{(0)}(x, y)=x, M^{(1)}(x, y)=y$ and, for $n \in \mathbb{N}_{0}$ and $0 \leq k \leq 2^{n+1}$,

$$
F^{\left(\frac{k}{2^{n+1}}\right)}(x, y)=\left\{\begin{array}{ll}
F^{\left(\frac{k}{2^{n}}\right)}(x, F(x, y)), & \text { if } 0 \leq k \leq 2^{n} \\
F^{\left(\frac{k-2^{n}}{2^{n}}\right)}(F(x, y), y), & \text { if } 2^{n} \leq k \leq 2^{n+1}
\end{array}, x, y \in I .\right.
$$

In spite of the resemblance among (15) and (5), the properties of $M^{[d]}$ and $M^{(d)}$ differ considerably each other. As it will be seen along the next sections, there is for Ryll-Nardzewski's dyadic iterations no result so simple as Theor. 1 and, to a certain extent, the independent study of $M^{[d]}$ conducted along this paper is justified by this fact. This study is structured as follows: in Section 2 are gather together the elementary properties of $M^{[d]}$, while the study of the continuity of the monotonic extensions of the map $d \mapsto M^{[d]}(x, y)$ is developed along the Sections 3, 4 and 5: the study of continuity at rational points (Section 3 ) is followed by the corresponding study at the irrationals of $[0,1]$ (Section 4 ), while a dynamic characterization of continuity is given at Section 5 . The comparatively complex behavior of the Ryll-Nardzewski's dyadic iterations is there explained. Some auxiliary results not devoid of intrinsic interest are gather together in the final Appendix.

\section{First properties of Ryll-Nardzewski's dyadic iterations}

In [8] and [4], the elementary properties of the Aczél's dyadic iterations of a function $F$ were collected. The corresponding study for Ryll-Nardzewski's dyadic iterations is abridged in this section. Let us begin with the following: 
Proposition 2 Let $F: I \times I \rightarrow I$ be a function. The following assertions concerning $F^{[d]}$ holds:

i) If $F$ is continuous or reflexive, then $F^{[d]}$ is, respectively, continuous or reflexive for every $d \in \operatorname{Dyad}([0,1])$;

ii) If $F$ is internal, then $F^{[d]}$ is internal for every $d \in \operatorname{Dyad}([0,1])$. Moreover, if $F$ is strictly internal, then $F^{[d]}$ is strictly internal for every $d \in$ $\operatorname{Dyad}([0,1]), d \neq 0,1$;

iii) If $F$ is isotone, then $F^{[d]}$ is isotone for every $d \in \operatorname{Dyad}([0,1])$. Moreover, if $F$ is strictly isotone, then $F^{[d]}$ is strictly isotone for every $d \in$ $\operatorname{Dyad}([0,1]), d \neq 0,1$;

iv) If $F$ is a symmetric function, then

$$
F^{[d]}(y, x)=F^{[1-d]}(x, y), x, y \in I,
$$

for every $d \in \operatorname{Dyad}([0,1])$.

Proof. The simple inductive proofs of the assertions will be, with exception of iv), omitted. For $d=0$ or $d=1$ the equality (16) is true by (4). Assume that it was true for all dyadic fractions with denominator $2^{n}$, then, (5) with $d=k / 2^{n+1}, 0 \leq k \leq 2^{n+1}$, and the symmetry of $F$ yield

$$
\begin{aligned}
& F^{\left[\frac{k}{2^{n+1}}\right]}(y, x)= \begin{cases}F\left(F^{\left[1-\frac{k}{2^{n}}\right]}(x, y), y\right), & \text { if } 0 \leq k \leq 2^{n} \\
F\left(x, F^{\left[1-\frac{h-2^{n}}{2^{n}}\right]}(x, y)\right), & \text { if } 2^{n} \leq k \leq 2^{n+1}\end{cases} \\
& =\left\{\begin{array}{ll}
F^{\left[\frac{1-\frac{k}{2^{n}+1}}{2}\right]}(x, y), \quad \text { if } 0 \leq k \leq 2^{n} \\
F\left[\frac{\left.1-\frac{h-2^{n}}{2^{n}}\right]}{F^{n}}(x, y), \quad \text { if } 2^{n} \leq k \leq 2^{n+1}\right.
\end{array}=F^{\left[1-\frac{k}{2^{n+1}}\right]}(x, y) .\right.
\end{aligned}
$$

This completes the inductive proof.

The simple proof of the following important result will be omitted.

Proposition 3 Let $f: I \rightarrow \mathbb{R}$ a bijective function; then $\left(F_{f}\right)^{[d]}=\left(F^{[d]}\right)_{f}$ for every $d \in \operatorname{Dyad}([0,1])$.

As a consequence, iterations and conjugacy commute each other and therefore, Ryll-Nardzewski's dyadic iterations of a homogeneous mean turn out to be homogeneous.

A function $F$ is said to be self-distributive or bisymmetric depending on equation (7) or (6) is satisfied by $F$. Clearly, a bisymmetric function is also a self-distributive function. Ryll-Nardzewski's dyadic iterations coincide with Aczél's ones on the family of self distributive functions.

Proposition 4 If $F$ is a self-distributive function, then $F^{[d]}=F^{(d)}$, for every $d \in \operatorname{Dyad}([0,1])$. 
Since quasiarithmetic means are bisymmetric functions, $\left(A_{f}\right)^{[d]}=\left(A_{f}\right)^{(d)}$ for every $d \in \operatorname{Dyad}([0,1])$, as asserted in the Introduction.

Proof. It is inductively proved that, if $F$ is a self-distributive function, then (5) can be replaced by $(15)$ and therefore, $F^{[d]}=F^{(d)}$, for every $d \in \operatorname{Dyad}([0,1])$. The details are omitted.

Remark 5 (On the proofs of Aczél's and Ryll-Nardzewski's theorems) The last result shown that Aczél's dyadic iterations can be replaced by Ryll-Nardzewski's ones in the proof of the characterization theorem of [1] and, correspondingly, that Aczél's dyadic iterations can be taken instead of Ryll-Nardzewski's ones in the characterization theorem of [22].

\section{Continuity at rational points}

In what follows, the study of the Ryll-Nardzewski's dyadic iterations will be circumscribed to class of means. For a general mean $M$, the behavior of these iterations is appreciably different from that of Aczél's ones. First of all, unlike the map $d \mapsto M^{(d)}(x, y)$, the map $d \mapsto M^{[d]}(x, y)$ is not necessarily strictly monotonic (for $x \neq y$ !) on the class of strict means. This fact is illustrated by the example below.

Example 6 (A mean with non monotonic iterations) The counter-harmonic mean $\mathrm{CH}$, defined by

$$
C H(x, y)=\frac{x^{2}+y^{2}}{x+y}, x, y>0
$$

is a symmetric continuous strict mean on $\mathbb{R}^{+}$. As a simple computation shows,

$$
C H^{\left[\frac{9}{16}\right]}(x, y)-C H^{\left[\frac{1}{2}\right]}(x, y)=C H(C H(x, C H(x, C H(x, y))), y)-C H(x, y)
$$

and, in view of the first term of the Taylor expansion of the function

$$
\delta \mapsto C H(C H(\delta, C H(\delta, C H(\delta, 2+\delta))), 2+\delta)-C H(\delta, 2+\delta)
$$

is given by

$$
C H(C H(\delta, C H(\delta, C H(\delta, 2+\delta))), 2+\delta)-C H(\delta, 2+\delta)=-\frac{1}{2} \delta+O\left(\delta^{2}\right),
$$

it is concluded that $C H^{\left[\frac{9}{16}\right]}(\delta, 2+\delta)-C H^{\left[\frac{1}{2}\right]}(\delta, 2+\delta)<0$ for every small enough $\delta>0$.

However, the iterations become monotonic when applied to an isotone mean.

Lemma 7 If $M$ is a [strictly] isotone mean, then the map Dyad $([0,1]) \ni d \mapsto$ $M^{[d]}(x, y)$ turns out to be [strictly] increasing when $x<y$ and [strictly] decreasing when $x>y$. 
In the above statement, Dyad $([0,1])$ is given the order induced by the usual order on $\mathbb{R}$. Of course, $M^{[d]}(x, x) \equiv x$ in $I$ for every $d \in \operatorname{Dyad}([0,1])$.

Proof. The simple inductive proof of this lemma will be omitted.

As a consequence of this lemma and from the fact that $\operatorname{Dyad}([0,1])$ is a dense subset of $[0,1]$, the map $d \mapsto M^{[d]}(x, y)$ can be extended to a [strictly] monotonic map defined on $[0,1]$ when $M$ is [strictly] isotone. In fact, for a given $\delta \in[0,1]$, consider the sets

$$
D^{-}(\delta)=\{d \in \operatorname{Dyad}([0,1]): d \leq \delta\}
$$

and

$$
D^{+}(\delta)=\{d \in \operatorname{Dyad}([0,1]): d \geq \delta\}
$$

then, the map defined for every $x, y \in I$ by

$$
M_{-}^{[\delta]}(x, y)= \begin{cases}\sup _{d \in D^{-}(\delta)} M^{[d]}(x, y), & \text { if } x<y \\ x, & \text { if } x=y \\ \inf _{d \in D^{-}(\delta)} M^{[d]}(x, y), & \text { if } x>y\end{cases}
$$

is the left continuous monotonic extension of $d \mapsto M_{-}^{[d]}(x, y)$. The right continuous monotonic extension $M_{+}^{[d]}(x, y)$ of $d \mapsto M^{[d]}(x, y)$ is similarly defined by substituting in (17) $\sup _{d \in D^{-}(\delta)}$ by $\inf _{d \in D^{+}(\delta)}$ and $\inf _{d \in D^{-}(\delta)}$ by $\sup _{d \in D^{+}(\delta)}$. Note that the equality

$$
M_{-}^{[\delta]}(x, y)=\lim _{n \uparrow+\infty} M^{\left[d_{n}\right]}(x, y)
$$

holds when $\left\{d_{n}: n \in \mathbb{N}\right\} \subseteq \operatorname{Dyad}([0,1])$ is any increasing sequence such that $d_{n} \uparrow \delta \in[0,1]$ when $n \uparrow+\infty$, and that, correspondingly, by taking decreasing sequences in $\operatorname{Dyad}([0,1])$ with $d_{n} \downarrow \delta \in[0,1]$ when $n \uparrow+\infty$, the equality

$$
M_{+}^{[d]}(x, y)=\lim _{n \uparrow+\infty} M^{\left[d_{n}\right]}(x, y)
$$

is obtained.

Now, for a [strictly] isotone mean $M$, the extended maps $\delta \mapsto M_{-}^{[\delta]}(x, y)$ and $\delta \mapsto M_{+}^{[\delta]}(x, y)$ are both [strictly] monotonic for $x \neq y$. For example, consider $\delta \mapsto M_{-}^{[\delta]}(x, y)$ for a strictly isotone mean $M$ and take $x<y$ and $\delta_{1}, \delta_{2} \in[0,1]$ such that $\delta_{1}<\delta_{2}$. Then there exist $d, d^{\prime} \in \operatorname{Dyad}([0,1])$ such that $\delta_{1}<d<d^{\prime}<\delta_{2}$; thus, Lemma 7 and (17) imply

$$
M_{-}^{\left[\delta_{1}\right]}(x, y) \leq M_{-}^{[d]}(x, y)<M_{-}^{\left[d^{\prime}\right]}(x, y) \leq M_{-}^{\left[\delta_{2}\right]}(x, y) .
$$

The case in which $x>y$ is similar, while $M_{-}^{[\delta]}(x, x) \equiv x$.

Clearly, the respectively left and right-continuous maps $\delta \mapsto M_{-}^{[\delta]}(x, y)$ and $\delta \mapsto M_{+}^{[\delta]}(x, y)$ have the same points of discontinuity. In this way, to study the continuity of the monotonic extensions of $d \mapsto M^{[d]}(x, y)$ it suffices to consider, 
as made in the remaining of this paper, the left continuous monotonic extension $\delta \mapsto M_{-}^{[\delta]}(x, y)$. As a first issue, the continuity of $\delta \mapsto M_{-}^{[\delta]}(x, y)$ at dyadic points of $[0,1]$ is established under the additional hypothesis of separated continuity of $M$.

Theorem 8 Let $M$ be a strictly isotone and separately continuous mean defined on $I$; then, for every $x, y \in I$, the following assertions hold:

i) 0 and 1 are points of continuity of $\delta \mapsto M_{-}^{[\delta]}(x, y)$;

ii) if $\xi \in[0,1]$ is a point of continuity of $\delta \mapsto M_{-}^{[\delta]}(x, y)$, then $\xi / 2$ and $(\xi+1) / 2$ are points of continuity as well;

iii) $\delta \mapsto M_{-}^{[\delta]}(x, y)$ is continuous on $\operatorname{Dyad}([0,1])$.

Proof. Clearly, $\delta \mapsto M_{-}^{[\delta]}(x, y)$ is continuous (by the left) at 1 . To show the continuity (by the right) at 0 , it suffices to prove that $M^{\left[1 / 2^{n}\right]}(x, y) \rightarrow x$ when $n \uparrow+\infty$. Indeed, the bounded sequence $\left\{M^{\left[1 / 2^{n}\right]}(x, y): n \in \mathbb{N}\right\}$ is increasing when $x<y$ and decreasing when $x>y$ (while $\left.M^{1 / 2^{n}}(x, x) \equiv x\right)$. Now, (5) yields

$$
M^{\left[1 / 2^{n+1}\right]}(x, y)=M\left(x, M^{\left[1 / 2^{n}\right]}(x, y)\right)
$$

so that, setting $\lim _{n \uparrow+\infty} M^{\left[1 / 2^{n}\right]}(x, y)=\mu \geq x$ and taking into account the continuity of $y \mapsto M(x, y)$, a passage to the limit in this equality gives

$$
\mu=M(x, \mu)
$$

whence, in view of the strict isotonicity of $M$, the equality $\mu=x$ follows.

The proof of ii) easily follows from (5). In fact, for $\delta \in[0,1]$ let us consider a sequence $\left(d_{n}: n \in \mathbb{N}\right) \subseteq \operatorname{Dyad}([0,1])$ such that $d_{n} \uparrow \delta$ when $n \uparrow+\infty$; then, by (5) it can be written

$$
M^{\left[\frac{d_{n}}{2}\right]}(x, y)=M\left(x, M^{\left[d_{n}\right]}(x, y)\right) \text { and } M^{\left[\frac{d_{n}+1}{2}\right]}(x, y)=M\left(M^{\left[d_{n}\right]}(x, y), y\right),
$$

whence (taking into account the separated continuity of $M$ ) a passage to the limit $n \uparrow+\infty$ yields

$$
M_{-}^{\left[\frac{\delta}{2}\right]}(x, y)=M\left(x, M_{-}^{[\delta]}(x, y)\right) \text { and } M_{-}^{\left[\frac{\delta+1}{2}\right]}(x, y)=M\left(M_{-}^{[\delta]}(x, y), y\right) .
$$

Now, if $\xi \in[0,1]$ is a point of continuity of $\delta \mapsto M_{-}^{[\delta]}(x, y)$ and $\left(d_{n}^{\prime}: n \in \mathbb{N}\right) \subseteq$ $\operatorname{Dyad}([0,1])$ is a sequence such that $d_{n}^{\prime} \downarrow \xi$ when $n \uparrow+\infty$, then (19) and the continuity of $M$ give

$$
\lim _{n \uparrow+\infty} M^{\left[\frac{d_{n}^{\prime}}{2}\right]}(x, y)=\lim _{n \uparrow+\infty} M\left(x, M^{\left[d_{n}^{\prime}\right]}(x, y)\right)=M\left(x, M_{-}^{[\xi]}(x, y)\right)=M_{-}^{\left[\frac{\xi}{2}\right]}(x, y)
$$


and, similarly,

$$
\lim _{n \uparrow+\infty} M^{\left[\frac{d_{n}^{\prime}+1}{2}\right]}(x, y)=\lim _{n \uparrow+\infty} M\left(M^{\left[d_{n}^{\prime}\right]}(x, y), y\right)=M\left(M_{-}^{[\xi]}(x, y), y\right)=M_{-}^{\left[\frac{\xi+1}{2}\right]}(x, y),
$$

which prove the continuity of $\delta \mapsto M_{-}^{[\delta]}(x, y)$ at $\xi / 2$ and $(\xi+1) / 2$, respectively.

iii) follows from i) and ii) by induction: $\delta \mapsto M_{-}^{[\delta]}(x, y)$ is continuous at $\delta=0,1$ and, if it was continuous at the points of the form $k / 2^{n}$ with $k=$ $0,1, \ldots, 2^{n}$, then it turns out to be continuous at the points of the form $k / 2^{n+1}$ with $k=0,1, \ldots, 2^{n+1}$. This completes the proof.

The conclusions of the above theorem are not maintained when strict isotonicity is replaced by isotonicity: coordinate or extremal means illustrate this fact.

As shown by the Example 14 below, the continuity of $\delta \mapsto M_{-}^{[\delta]}(x, y)$ at a rational point $r \notin \operatorname{Dyad}([0,1])$ can not be generally ensured under the hypotheses of Theor. 8. In the following paragraphs, a series of concepts is introduced to facilitate the study of the continuity at such points.

In the first place, recall that a dyadic rational $\varepsilon \in \operatorname{Dyad}([0,1]), \varepsilon \neq 1$, admits a finite binary representation of the form $\left(0 . \varepsilon_{1} \cdots \varepsilon_{n}\right)_{2}$ with $\varepsilon_{i} \in\{0,1\}$, $i=1, \ldots, n$, and $\varepsilon_{n}=1$. In general, a rational number $\xi \in[0,1]$ has a binary expansion of the form

$$
\xi=\left(0 . \xi_{1} \cdots \xi_{n} \overline{\varepsilon_{1} \cdots \varepsilon_{p}}\right)_{2},
$$

where $\xi_{1} \cdots \xi_{n}$ is the non-periodic part while $\overline{\varepsilon_{1} \cdots \varepsilon_{p}}$ denotes the periodic part of the expansion. The finite sequence of digits $\varepsilon_{1} \cdots \varepsilon_{p}$ is usually named period of the expansion and it is understood that it is minimal; i.e., there is no finite sequence of digits $\varepsilon_{1} \cdots \varepsilon_{p^{\prime}}$ with $p^{\prime}<p$ such that $\xi=\left(0 . \xi_{1} \cdots \xi_{n} \overline{\varepsilon_{1} \cdots \varepsilon_{p^{\prime}}}\right)_{2}$. A rational $\xi \in[0,1]$ is said to be a periodic dyadic fraction when its binary expansion (20) does not contain a non-periodic part; i.e., when the binary expansion of $\xi$ has the form $\xi=\left(0 . \overline{\varepsilon_{1} \cdots \varepsilon_{p}}\right)_{2}$.

The set of all finite sequences of digits will be denoted by $\mathcal{F S}\{0,1\}$; i.e. $\mathcal{F S}\{0,1\}=\bigcup_{n \in \mathbb{N}}\{0,1\}^{n}$. The length of the sequence $\varepsilon=\left(\varepsilon_{1}, \ldots, \varepsilon_{n}\right)$ is $\ell(\varepsilon)=$ $n$. On $\mathcal{F} \mathcal{S}\{0,1\}$ it is defined the operation ' $\&$ ' (concatenation) by

$$
\left(\varepsilon_{1}, \ldots, \varepsilon_{m}\right) \&\left(\varepsilon_{1}^{\prime}, \ldots, \varepsilon_{n}^{\prime}\right)=\left(\varepsilon_{1}, \ldots, \varepsilon_{m}, \varepsilon_{1}^{\prime}, \ldots, \varepsilon_{n}^{\prime}\right) .
$$

If $\left(\varepsilon_{1}, \ldots, \varepsilon_{p}\right) \in \mathcal{F S}\{0,1\}$ is of form $\left(\varepsilon_{1}, \ldots, \varepsilon_{p}\right)=\left(\varepsilon_{1}, \ldots, \varepsilon_{q}\right) \& \cdots \&\left(\varepsilon_{1}, \ldots, \varepsilon_{q}\right)$ for a certain divisor $q$ of $p$, then $\left(\varepsilon_{1}, \ldots, \varepsilon_{p}\right)$ is said to be a repeating sequence. Thus, the sequence of digits $\left(\varepsilon_{1}, \ldots, \varepsilon_{q}\right)$ corresponding to the periodic part of the binary expansion (20) is not a repeating sequence. An important role will correspond to the subset of $\mathcal{F S}\{0,1\}$ constituted by all non repeating sequences, so that it will be denoted by $\mathcal{N} \mathcal{R} \mathcal{F} \mathcal{S}\{0,1\}$.

The sets $\{0,1\}^{n}, n \in \mathbb{N}$, will be equipped with the lexicographic order induced by the order $\preceq$ defined on $\{0,1\}$ by $\{(0,0),(0,1),(1,1)\}$. Recall that this order is defined by $\left(\varepsilon_{1}, \ldots, \varepsilon_{n}\right) \preceq\left(\varepsilon_{1}^{\prime}, \ldots, \varepsilon_{n}^{\prime}\right)$ if $\varepsilon_{i} \preceq \varepsilon_{i}^{\prime}$ where $i=\min \left\{j: \varepsilon_{j} \neq\right.$ $\left.\varepsilon_{j}^{\prime}\right\}$. If $\left(0 . \varepsilon_{1} \cdots \varepsilon_{n}\right)_{2}$ is a dyadic fraction, then $\left(0 . \varepsilon_{1} \cdots \varepsilon_{n}\right)_{2}=\left(0 . \varepsilon_{1} \cdots \varepsilon_{n} 0 \cdots 0\right)_{2}$ so that, by eventually adding zeros to any expansion, the length of two dyadic 
fractions $\left(0 . \varepsilon_{1} \cdots \varepsilon_{m}\right)_{2}$ and $\left(0 . \varepsilon_{1}^{\prime} \cdots \varepsilon_{n}^{\prime}\right)_{2}$ can be matched. Take, for instance, $m<n$; then, the inequality $\left(0 . \varepsilon_{1} \cdots \varepsilon_{m}\right)_{2} \leq\left(0 . \varepsilon_{1}^{\prime} \cdots \varepsilon_{n}^{\prime}\right)_{2}$ holds if and only if $\left(\varepsilon_{1}, \ldots, \varepsilon_{m}, 0, \ldots, 0\right) \preceq\left(\varepsilon_{1}^{\prime}, \ldots, \varepsilon_{n}^{\prime}\right)$, where $m-n$ zeros have been added to the expansion $\left(0 . \varepsilon_{1} \cdots \varepsilon_{m}\right)_{2}$.

Now, let us associate to a finite sequence of digits $\left(\varepsilon_{1}, \ldots, \varepsilon_{n}\right) \in \mathcal{F} \mathcal{S}\{0,1\}$ the map $h_{(\varepsilon)}:[0,1] \rightarrow[0,1]$ given by

$$
h_{(\varepsilon)}(t)=\left(h_{\varepsilon_{1}} \circ \cdots \circ h_{\varepsilon_{n}}\right)(t), t \in[0,1]
$$

where

$$
h_{\varepsilon_{i}}(t)=\left\{\begin{array}{cc}
\frac{t}{2}, & \text { if } \varepsilon_{i}=0 \\
\frac{t+1}{2}, & \text { if } \varepsilon_{i}=1
\end{array}, t \in[0,1] .\right.
$$

Clearly, the equality

$$
h_{\left(\varepsilon \& \varepsilon^{\prime}\right)}=h_{(\varepsilon)} \circ h_{\left(\varepsilon^{\prime}\right)}
$$

holds for every $\varepsilon, \varepsilon^{\prime} \in \mathcal{F S}\{0,1\}$. It should be noted that, if $\left(\varepsilon_{1}, \ldots, \varepsilon_{n}\right) \in$ $\{0,1\}^{n}$ and $\varepsilon_{n}=1$, then $\left(0 . \varepsilon_{1} \cdots \varepsilon_{n}\right)_{2}$ is the binary expansion of $\varepsilon=k / 2^{n} \in$ $\operatorname{Dyad}([0,1])$, where $k=\sum_{i=1}^{n} \varepsilon_{i} 2^{n-i}$. In view of this fact, for $\varepsilon \in \operatorname{Dyad}([0,1]), \varepsilon \neq$ 1 , let us define $h_{(\varepsilon)}$ also by (21)-(22) provided that $\varepsilon=\left(0 . \varepsilon_{1} \cdots \varepsilon_{n}\right)_{2}$.

Lemma 9 If $\varepsilon=\left(\varepsilon_{1}, \ldots, \varepsilon_{n}\right) \in \mathcal{F} \mathcal{S}\{0,1\}$, then

$$
h_{(\varepsilon)}(t)=\frac{t+k}{2^{n}}, t \in[0,1],
$$

where $k=\sum_{i=1}^{n} \varepsilon_{i} 2^{n-i}$. In particular, if $\varepsilon=\left(0 . \varepsilon_{1}, \ldots, \varepsilon_{n}\right)_{2} \in \operatorname{Dyad}([0,1]), \varepsilon \neq$ 1 , then

$$
h_{(\varepsilon)}(t)=\frac{t}{2^{n}}+\varepsilon, t \in[0,1] .
$$

Proof. (23) is true for $\varepsilon=(0)$ and $\varepsilon=(1)$. Assuming that it was true for a given $n \geq 1$ and every $\varepsilon=\left(\varepsilon_{1}, \ldots, \varepsilon_{n}\right) \in\{0,1\}^{n}$, then (21) yields

$$
\begin{aligned}
h_{\left(\varepsilon_{1}, \ldots, \varepsilon_{n}, \varepsilon_{n+1}\right)}(t) & =h_{\left(\varepsilon_{1}, \ldots, \varepsilon_{n}\right)}\left(h_{\varepsilon_{n+1}}(t)\right)= \begin{cases}\frac{\frac{t}{2}+\sum_{i=1}^{n} \varepsilon_{i} 2^{n-i}}{2^{n}}, & \text { if } \varepsilon_{n+1}=0 \\
\frac{\frac{t+1}{2}+\sum_{i=1}^{n} \varepsilon_{i} 2^{n-i}}{2^{n}}, & \text { if } \varepsilon_{n+1}=1\end{cases} \\
& =\frac{t+k}{2^{n+1}},
\end{aligned}
$$

where $k=\sum_{i=1}^{n+1} \varepsilon_{i} 2^{n+1-i}$. This finishes the inductive proof of (23). Now, if $\varepsilon=\left(0 . \varepsilon_{1}, \ldots, \varepsilon_{n}\right)_{2} \in \operatorname{Dyad}([0,1]), \varepsilon \neq 1$, then $\varepsilon=k / 2^{n}$ with $k=\sum_{i=1}^{n} \varepsilon_{i} 2^{n-i}$ and (24) follows from (23).

Suppose that $M$ is a mean and fix a point $(x, y) \in I^{2}$. Denote by $I_{(x, y)}$ the interval $[\min \{x, y\}, \max \{x, y\}]$. As made before with the maps $h_{(\varepsilon)}$, for every $\varepsilon=\left(\varepsilon_{1}, \ldots, \varepsilon_{n}\right) \in \mathcal{F S}\{0,1\}$ let us define a map $m_{(\varepsilon)}: I_{(x, y)} \rightarrow I_{(x, y)}$ by

$$
m_{(\varepsilon)}(u)=\left(m_{\varepsilon_{1}} \circ \cdots \circ m_{\varepsilon_{n}}\right)(u), u \in I_{(x, y)},
$$

where

$$
m_{\varepsilon_{i}}(u)=\left\{\begin{array}{ll}
M(x, u), & \text { if } \varepsilon_{i}=0 \\
M(u, y), & \text { if } \varepsilon_{i}=1
\end{array}, u \in I_{(x, y)} .\right.
$$


Note that, when $x=y, m_{(\varepsilon)}$ is defined on the unique point $u=x$ and $m_{(\varepsilon)}(x)=$ $x$. The equality

$$
m_{\left(\varepsilon \& \varepsilon^{\prime}\right)}=m_{(\varepsilon)} \circ m_{\left(\varepsilon^{\prime}\right)}
$$

holds for every pair of sequences $\varepsilon, \varepsilon^{\prime} \in \mathcal{F} \mathcal{S}\{0,1\}$.

If $\varepsilon=\left(0 . \varepsilon_{1} \cdots \varepsilon_{n}\right)_{2} \in \operatorname{Dyad}([0,1]), m_{(\varepsilon)}$ is also defined by (25)-(26). Even if the maps $m_{(\varepsilon)}$ depend on the point $(x, y) \in I^{2}$, this dependence will turn out to be not relevant in the next developments.

In the next result, the main properties of the maps $m_{(\varepsilon)}$ are established.

Proposition 10 Let $M$ be a mean defined on I, then, the following assertions hold:

i) if $M$ is separately continuous, then $m_{(\varepsilon)}, \varepsilon \in \mathcal{F} \mathcal{S}\{0,1\}$, is continuous on $I_{(x, y)}$

ii) if $M$ is [strictly] isotone, then $m_{(\varepsilon)}, \varepsilon \in \mathcal{F} \mathcal{S}\{0,1\}$, is [strictly] increasing on $I_{(x, y)}$ when $x \neq y$; moreover,

iii) if $\varepsilon, \varepsilon^{\prime} \in\{0,1\}^{n}$, then

$$
m_{(\varepsilon)}(u) \leq m_{\left(\varepsilon^{\prime}\right)}(u), u \in I_{(x, y)},
$$

provided that $\varepsilon \preceq \varepsilon^{\prime}$ and $x<y$; the inequality (27) is reversed when $x>y$. In particular, if $\varepsilon, \varepsilon^{\prime} \in \operatorname{Dyad}([0,1]), \varepsilon \neq 1 \neq \varepsilon^{\prime}$, then $m_{(\varepsilon)} \leq m_{\left(\varepsilon^{\prime}\right)}$ in $I_{(x, y)}$ provided that $\varepsilon \leq \varepsilon^{\prime}$ and $x<y$ (while $m_{\left(\varepsilon^{\prime}\right)} \leq m_{(\varepsilon)}$ in $I_{(x, y)}$ when $x>y$ ).

Proof. i) follows from (25)-(26) and the separated continuity of $M$.

Since $m_{0}(u)=M(x, u)$ and $m_{1}(u)=M(u, y)$ are [strictly] increasing functions on $I_{(x, y)}$ by the [strict] isotonicity of $M$, the composition of a certain number of functions chosen among $m_{0}$ and $m_{1}$ turns out to be [strictly] increasing as well. This proves ii).

Regarding iii), observe that

$$
m_{0}(u) \leq M(x, y) \leq m_{1}(u)
$$

for every $u \in I_{(x, y)}$ when $x<y$, while the inequalities (28) are reversed when $x>y$. Thus, if $\varepsilon=\left(\varepsilon_{1}, \ldots, \varepsilon_{n}\right) \preceq\left(\varepsilon_{1}^{\prime}, \ldots, \varepsilon_{n}^{\prime}\right)=\varepsilon^{\prime}$ and $x<y$, then $\varepsilon=$ $\left(\varepsilon_{1}, \ldots, \varepsilon_{i-1}, 0, \varepsilon_{i+1} \ldots, \varepsilon_{n}\right)$ and $\left(\varepsilon_{1}, \ldots, \varepsilon_{i-1}, 1, \varepsilon_{i+1}^{\prime}, \ldots, \varepsilon_{n}^{\prime}\right)=\varepsilon^{\prime}$, whence, in view of ii),

$$
\begin{aligned}
m_{(\varepsilon)}(u) & =m_{\left(\varepsilon_{1}, \ldots, \varepsilon_{i-1}\right)}\left(m_{0}\left(m_{\left(\varepsilon_{i+1} \ldots, \varepsilon_{n}\right)}(u)\right)\right) \\
& \leq m_{\left(\varepsilon_{1}, \ldots, \varepsilon_{i-1}\right)}\left(m_{1}\left(m_{\left(\varepsilon_{i+1}^{\prime}, \ldots, \varepsilon_{n}^{\prime}\right)}(u)\right)\right)=m_{\left(\varepsilon^{\prime}\right)}(u)
\end{aligned}
$$

for every $u \in I_{(x, y)}$. Clearly, (29) holds in the opposite sense when $x>y$. The proof in the case in which $\varepsilon, \varepsilon^{\prime} \in \operatorname{Dyad}([0,1])$ is derived from this and the discussion preceding Lemma 9.

A relationship existing among the maps $h_{(\varepsilon)}$ and $m_{(\varepsilon)}$ and some of its consequences are established by the following result. The set of fixed points of a map $f$ is to be denoted by $\operatorname{Fix}(f)$. 
Proposition 11 Let $M$ be a mean defined on I; then, the following asseverations hold:

i) if $\varepsilon \in \mathcal{F} \mathcal{S}\{0,1\}$ and $d \in \operatorname{Dyad}([0,1]), d \neq 1$, then

$$
M^{\left[h_{(\varepsilon)}(d)\right]}(x, y)=m_{(\varepsilon)}\left(M^{[d]}(x, y)\right), x, y \in I ;
$$

ii) if $\varepsilon=\left(\varepsilon_{1}, \ldots, \varepsilon_{n}\right) \in \mathcal{N} \mathcal{R} \mathcal{F} \mathcal{S}\{0,1\}$, then

$$
m_{(\varepsilon)}(x)=M^{\left[\frac{k}{2^{n}}\right]}(x, y), x, y \in I,
$$

and

$$
m_{(\varepsilon)}(y)=M^{\left[\frac{k+1}{2^{n}}\right]}(x, y), x, y \in I,
$$

where $k=\sum_{i=1}^{n} \varepsilon_{i} 2^{n-i} ;$

iii) $\operatorname{Fix}\left(m_{(\varepsilon)}\right) \neq \emptyset$ for every $\varepsilon \in \mathcal{F} \mathcal{S}\{0,1\}$ provided that $M$ is separately continuous; furthermore, if $M$ is strictly isotone, then

$$
\operatorname{Fix}\left(m_{(\varepsilon)}\right) \subseteq(\min \{x, y\}, \max \{x, y\}) ;
$$

iv) when $M$ is strictly isotone and separately continuous, $\delta \mapsto M_{-}^{[\delta]}(x, y)$ is continuous on the whole interval $[0,1]$ if and only if it is continuous on a subinterval $\emptyset \neq(a, b) \subseteq[0,1]$.

Note that the equalities

$$
M^{\left[h_{(\varepsilon)}^{k}(d)\right]}(x, y)=m_{(\varepsilon)}^{k}\left(M^{[d]}(x, y)\right), k \in \mathbb{N},
$$

are derived by iterating the equality (30) of assertion i). On its part, when $M$ is strictly isotone and separately continuous, the set of discontinuities of the map $\delta \mapsto M_{-}^{[\delta]}(x, y)$ is dense in $[0,1]$ provided that it is non void. This is a restatement of the assertion iv).

Proof. Let us prove i) by induction on the length $\ell(\varepsilon)$. If $\ell(\varepsilon)=1$ and $d \in \operatorname{Dyad}([0,1]), d \neq 1$, then $(22),(5)$ and $(26)$ yield

$$
\begin{aligned}
M^{\left[h_{\left(\varepsilon_{1}\right)}(d)\right]}(x, y) & = \begin{cases}M^{\left[\frac{d}{2}\right]}(x, y), & \text { if } \varepsilon_{1}=0 \\
M^{\left[\frac{d+1}{2}\right]}(x, y), & \text { if } \varepsilon_{1}=1\end{cases} \\
& = \begin{cases}M\left(x, M^{[d]}(x, y)\right), & \text { if } \varepsilon_{1}=0 \\
M\left(M^{[d]}(x, y), y\right), & \text { if } \varepsilon_{1}=1\end{cases} \\
& =m_{\left(\varepsilon_{1}\right)}\left(M^{[d]}(x, y)\right), x, y \in I .
\end{aligned}
$$

Thus, assuming that (30) was true for $\ell(\varepsilon)=n \geq 1$ and every $d \in \operatorname{Dyad}([0,1]), d \neq$ 1 , for $\varepsilon \in \mathcal{F} \mathcal{S}\{0,1\}$ with $\ell(\varepsilon)=n+1$, it can be written $\varepsilon=\varepsilon^{\prime} \&\left(\varepsilon_{n+1}\right)$ with 
$\ell\left(\varepsilon^{\prime}\right)=n$ and thus,

$$
\begin{aligned}
M^{\left[h_{(\varepsilon)}(d)\right]}(x, y) & =M^{\left[h_{\left(\varepsilon^{\prime} \&\left(\varepsilon_{n+1}\right)\right.}(d)\right]}(x, y) \\
& =M^{\left[h_{\left(\varepsilon^{\prime}\right)}\left(h_{\left(\varepsilon_{n+1}\right)}(d)\right)\right]}(x, y) \\
& =m_{\left(\varepsilon^{\prime}\right)}\left(M^{\left[h_{\left(\varepsilon_{n+1}\right)}(d)\right]}(x, y)\right) \\
& =m_{\left(\varepsilon^{\prime}\right)}\left(m_{\left(\varepsilon_{n+1}\right)}\left(M^{[d)]}(x, y)\right)\right) \\
& =\left(m_{\left(\varepsilon^{\prime}\right)} \circ m_{\left(\varepsilon_{n+1}\right)}\right)\left(M^{[d)]}(x, y)\right) \\
& =m_{\left(\varepsilon^{\prime} \&\left(\varepsilon_{n+1}\right)\right)}\left(M^{[d)]}(x, y)\right), x, y \in I,
\end{aligned}
$$

which completes the inductive proof of $\mathbf{i}$ ).

Setting $d=0$ in (30) yields

$$
M^{\left[h_{(\varepsilon)}(0)\right]}(x, y)=m_{(\varepsilon)}\left(M^{[0]}(x, y)\right)=m_{(\varepsilon)}(x), x, y \in I,
$$

whence, taking into account that $h_{(\varepsilon)}(0)=k / 2^{n}$ by Lemma 9 , equality (31) is obtained. By replacing $d=1$ in (30), a similar reasoning shows that (32) holds. This finishes the proof of assertion ii).

Regarding to iii), observe that the map $m_{(\varepsilon)}: I_{(x, y)} \rightarrow I_{(x, y)}$ is continuous by Prop. 10-i); thus, it has a fixed point at least. Now, if $M$ is strictly isotone and $\varepsilon=\left(\varepsilon_{1}, \ldots, \varepsilon_{n}\right) \in \mathcal{N} \mathcal{R} \mathcal{F} \mathcal{S}\{0,1\}$, then $\varepsilon \neq(0, \ldots, 0)$ and $\varepsilon \neq(1, \ldots, 1)$; thus, $0<k=\sum_{i=1}^{n} \varepsilon_{i} 2^{n-i}<1-1 / 2^{n}$ and, for $x<y$, the equalities proved in ii) yield

$$
\begin{aligned}
& x-m_{(\varepsilon)}(x)=x-M^{\left[\frac{k}{2^{n}}\right]}(x, y)<0, \\
& y-m_{(\varepsilon)}(y)=y-M^{\left[\frac{k+1}{2^{n}}\right]}(x, y)>0 .
\end{aligned}
$$

In this way, the extreme points of the interval $I_{(x, y)}$ are not fixed point of $m_{(\varepsilon)}$. These inequalities are reversed when $x>y$, so that the conclusion is maintained.

To prove iv), assume that $M$ is strictly isotone and separately continuous and fix $x, y \in I$. Replacing $d$ in (30) by the terms of an increasing sequence $\left(d_{n}: n \in \mathbb{N}\right)$ such that $d_{n} \uparrow \delta \in[0,1]$, it is obtained

$$
M^{\left[h_{(\varepsilon)}\left(d_{n}\right)\right]}(x, y)=m_{(\varepsilon)}\left(M^{\left[d_{n}\right]}(x, y)\right), n \in \mathbb{N},
$$

whence, passing to the limit $n \uparrow+\infty$, it is deduced

$$
M_{-}^{\left[h_{(\varepsilon)}(\delta)\right]}(x, y)=m_{(\varepsilon)}\left(M_{-}^{[\delta]}(x, y)\right),
$$

or, equivalently,

$$
m_{(\varepsilon)}^{-1}\left(M_{-}^{\left[h_{(\varepsilon)}(\delta)\right]}(x, y)\right)=M_{-}^{[\delta]}(x, y),
$$

since in view of Prop. 10-i) and ii) an inverse $m_{(\varepsilon)}^{-1}$ is admitted by $m_{(\varepsilon)}$. Observe that this last equality holds also for every $\varepsilon \in \operatorname{Dyad}([0,1]), \varepsilon \neq 1$. Now, assume 
that $\delta \mapsto M_{-}^{[\delta]}(x, y)$ is continuous on a subinterval $(a, b) \subseteq[0,1]$. There is no loss of generality in supposing that $(a, b)$ is of the form $\left(k / 2^{n},(k+1) / 2^{n}\right)$ for a certain $0<k<2^{n}-1$, so that choosing $\varepsilon=k / 2^{n}$, it turns out to be

$$
h_{(\varepsilon)}(\delta)=\frac{k+\delta}{2^{n}} \in\left(k / 2^{n},(k+1) / 2^{n}\right)
$$

for every $\delta \in(0,1)$ and therefore, the map $\delta \mapsto m_{(\varepsilon)}^{-1}\left(M_{-}^{\left[h_{(\varepsilon)}(\delta)\right]}(x, y)\right)$ is continuous at every point $\delta \in(0,1)$. This fact and (35) prove that $\delta \mapsto M_{-}^{[\delta]}(x, y)$ is continuous on $(0,1)$. The continuity at $\delta=0$ and $\delta=1$ was proved in Theor. 8 , so that $\delta \mapsto M_{-}^{[\delta]}(x, y)$ is continuous on the whole interval $[0,1]$. Since the converse is immediate, this concludes the proof of iv).

The extreme points of $\operatorname{Fix}\left(m_{(\varepsilon)}\right)$ when $\varepsilon \in \mathcal{N} \mathcal{R} \mathcal{F} \mathcal{S}\{0,1\}$ are characterized by the following:

Proposition 12 Let $M$ be a strictly isotone and separately continuous mean and $\bar{\varepsilon}=\left(0 . \overline{\varepsilon_{1} \cdots \varepsilon_{p}}\right)$ a periodic dyadic fraction. Then, $\varepsilon=\left(\varepsilon_{1}, \ldots, \varepsilon_{p}\right) \in$ $\mathcal{N} \mathcal{R} \mathcal{F S}\{0,1\}$ and $u_{0}=M_{-}^{[\bar{\varepsilon}]}(x, y)$ turns out to be a fixed point of $m_{(\varepsilon)}$. Furthermore, $M_{-}^{[\bar{\varepsilon}]}(x, y)$ is the leftmost point of $\operatorname{Fix}\left(m_{(\varepsilon)}\right)$ when $x<y$, while it is the rightmost one when $x>y$.

Proof. Define, for every $k \in \mathbb{N}_{0}, \bar{\varepsilon}_{k}=\varepsilon\left(1+1 / 2^{p}+\cdots+1 / 2^{k p}\right)$; then, $\bar{\varepsilon}_{k} \uparrow \bar{\varepsilon}$ when $k \uparrow+\infty$, and therefore,

$$
M_{-}^{[\bar{\varepsilon}]}(x, y)=\lim _{k \uparrow+\infty} M_{-}^{\left[\bar{\varepsilon}_{k}\right]}(x, y)
$$

On the other hand, by Prop. 10, it can be written

$$
M^{\left[\bar{\varepsilon}_{k}\right]}(x, y)=\overbrace{\left(m_{\varepsilon_{1}} \circ \cdots \circ m_{\varepsilon_{p}}\right) \circ \cdots \circ\left(m_{\varepsilon_{1}} \circ \cdots \circ m_{\varepsilon_{p}}\right)}^{k \text { times }}(x)=m_{(\varepsilon)}^{k}(x),
$$

and the limit $\lim _{k \uparrow+\infty} m_{(\varepsilon)}^{k}(x)$ (given by $(36)$ ) is a fixed point of the map $m_{\varepsilon}$. Furthermore, if $x<y$ and $u_{0}$ is the leftmost point of $\operatorname{Fix}\left(m_{(\varepsilon)}\right)$, then $u<$ $m_{\varepsilon}(u)<u_{0}$ for $x \leq u<u_{0}$; thus $m_{\varepsilon}^{k}(x) \uparrow u_{0}$ when $k \uparrow+\infty$. Now, if $x>y$ and $u_{0}$ is the rightmost point of $\operatorname{Fix}\left(m_{(\varepsilon)}\right)$, then $u_{0}<m_{\varepsilon}(u)<u$ for $u_{0}<u<x$ and therefore $m_{\varepsilon}^{k}(x) \downarrow u_{0}$ when $k \uparrow+\infty$. This finishes the proof.

For a rational point $\xi \in[0,1)$ with binary expansion given by $(20)$, it can be written

$$
M_{-}^{[\xi]}(x, y)=m_{\left(\xi_{1}, \ldots, \xi_{n}\right)}\left(M_{-}^{[\bar{\varepsilon}]}(x, y)\right),
$$

where $\bar{\varepsilon}=0 . \overline{\varepsilon_{1} \cdots \varepsilon_{p}}$. In fact, $\xi=\left(0 . \xi_{1} \cdots \xi_{n} \overline{\varepsilon_{1} \cdots \varepsilon_{p}}\right)_{2}=\left(0 . \xi_{1} \cdots \xi_{n}\right)_{2}+$ $\frac{1}{2^{n}}\left(0 . \overline{\varepsilon_{1} \cdots \varepsilon_{p}}\right)_{2}=h_{\left(\xi_{1}, \ldots, \xi_{n}\right)}\left(0 . \overline{\varepsilon_{1} \cdots \varepsilon_{p}}\right)$ and the equality (34) yields

$$
\begin{aligned}
M_{-}^{[\xi]}(x, y) & =M_{-}^{\left[h_{\left(\xi_{1}, \ldots, \xi_{n}\right)}(\bar{\varepsilon})\right]}(x, y) \\
& =m_{\left(\xi_{1}, \ldots, \xi_{n}\right)}\left(M_{-}^{[\bar{\varepsilon}]}(x, y)\right) .
\end{aligned}
$$


As a consequence of (38), the continuity of the map $\delta \mapsto M_{-}^{[\delta]}(x, y)$ at the rational point $\xi$ will depend on the dynamical properties of the map $m_{(\varepsilon)}$. This is the content of the following:

Theorem 13 Let $M$ be a strictly isotone and separately continuous mean defined on I. A necessary and sufficient condition in order that $\delta \mapsto M_{-}^{[\delta]}(x, y)$ be continuous at the rational point $\xi=\left(0 . \xi_{1} \cdots \xi_{n} \overline{\varepsilon_{1} \cdots \varepsilon_{p}}\right)_{2}$ is that the map $m_{(\varepsilon)}$ would have a unique fixed point in $(\min \{x, y\}, \max \{x, y\})$.

In this way, $\delta \mapsto M_{-}^{[\delta]}(x, y)$ is continuous on $[0,1] \cap \mathbb{Q}$ if and only if, for every $\varepsilon \in \mathcal{N} \mathcal{R} \mathcal{F} \mathcal{S}\{0,1\}$, the map $m_{(\varepsilon)}$ has a unique fixed point.

Proof. Let $\xi=\left(0 . \xi_{1} \cdots \xi_{n} \frac{\left(\varepsilon \varepsilon_{1} \cdots \varepsilon_{p}\right.}{\varepsilon_{2}}\right)_{2}$ be a rational number belonging to the interval $(0,1)$. To prove the sufficiency, fix a point $(x, y) \in I^{2}$ and assume that $m_{(\varepsilon)}, \varepsilon=\left(\varepsilon_{1}, \ldots, \varepsilon_{p}\right) \in \mathcal{N} \mathcal{R} \mathcal{F} \mathcal{S}\{0,1\}$, has in $(\min \{x, y\}, \max \{x, y\})$ a unique fixed point $u_{0}$ which, after Lemma 12 , is given by $u_{0}=M_{-}^{[\bar{\varepsilon}]}(x, y)$. Let us prove that $\delta \mapsto M_{-}^{[\delta]}(x, y)$ is continuous at $\bar{\varepsilon}$. First of all, observe that $0<\bar{\varepsilon}<1$ and therefore, for a small enough $\delta>0$, there exists $d_{0} \in \operatorname{Dyad}([0,1])$ such that $\bar{\varepsilon}<d_{0}<\bar{\varepsilon}+\delta$. The decreasing sequence $\left\{h_{(\varepsilon)}^{m}\left(d_{0}\right): m \in \mathbb{N}_{0}\right\}$ satisfies

$$
h_{(\varepsilon)}^{m}\left(d_{0}\right) \downarrow \bar{\varepsilon}
$$

when $m \uparrow+\infty$. In fact, by Lemma $9, h_{(\varepsilon)}\left(t_{0}\right)=t_{0}$ if and only if

$$
\frac{t_{0}+k}{2^{p}}=t_{0}
$$

where $k=\sum_{i=1}^{p} \varepsilon_{i} 2^{p-i} ;$ hence, the unique fixed point $t_{0}$ of $h_{(\varepsilon)}$ is given by

$$
t_{0}=\frac{k}{2^{p}-1}=\frac{\sum_{i=1}^{p} \varepsilon_{i} 2^{p-i}}{2^{p}-1}=\bar{\varepsilon}
$$

and therefore, $h_{(\varepsilon)}^{k}\left(d_{0}\right) \downarrow \bar{\varepsilon}$ when $k \uparrow+\infty$, as affirmed. On the other hand, $\lim _{k \uparrow+\infty} m_{(\varepsilon)}^{k}\left(M^{\left[d_{0}\right]}(x, y)\right)=u_{0}=M_{-}^{[\bar{\varepsilon}]}(x, y)$, and then (33) implies

$$
\lim _{k \uparrow+\infty} M^{\left[h_{(\varepsilon)}^{k}\left(d_{0}\right)\right]}(x, y)=\lim _{k \uparrow+\infty} m_{(\varepsilon)}^{k}\left(M^{\left[d_{0}\right]}(x, y)\right)=M_{-}^{[\bar{\varepsilon}]}(x, y),
$$

or, in view of (39),

$$
M_{+}^{[\bar{\varepsilon}]}(x, y)=M_{-}^{[\bar{\varepsilon}]}(x, y),
$$

i.e., $\delta \mapsto M_{-}^{[\delta]}(x, y)$ is continuous at $\bar{\varepsilon}$.

Now, the continuity of $\delta \mapsto M_{-}^{[\delta]}(x, y)$ at $\delta=\xi$ follows from (38) and the continuity at $\delta=\bar{\varepsilon}$.

To prove the converse, assume that $\operatorname{Fix}\left(m_{(\varepsilon)}\right)$ was not a singleton for a certain point $(x, y) \in I^{2}$. Then, assuming $x<y$, the sequence $\left\{M^{\left[\bar{\varepsilon}_{k}\right]}(x, y)\right\}$ where $\bar{\varepsilon}_{k}$ is defined like in the proof of Lemma 12 satisfies

$$
M_{-}^{[\bar{\varepsilon}]}(x, y)=\lim _{k \uparrow+\infty} M^{\left[\bar{\varepsilon}_{k}\right]}(x, y)=u_{1}
$$


where $u_{1}$ is the leftmost fixed point of $m_{(\varepsilon)}$. On the other hand, the sequence $\left(M^{\left[h_{(\varepsilon)}^{k}\left(d_{0}\right)\right]}(x, y): k \in \mathbb{N}\right)$ with $d_{0}$ defined as before satisfies

$$
M_{+}^{[\bar{\varepsilon}]}(x, y)=\lim _{k \uparrow+\infty} M^{h_{(\varepsilon)}^{k}\left(d_{0}\right)}(x, y)=u_{2}
$$

where $u_{2}$ is the rightmost fixed point of $m_{(\varepsilon)}$. Since $u_{1} \neq u_{2}, \delta \mapsto M_{-}^{[\delta]}(x, y)$ is not continuous at $\delta=\bar{\varepsilon}$ and, in view of (38), neither at $\delta=\xi$.

As a corollary of the proof of Theor. 13, at a rational point $\xi=\left(0 . \xi_{1} \cdots \xi_{n} \overline{\varepsilon_{1} \cdots \varepsilon_{p}}\right)_{2}$ it can be written

$$
\left[M_{-}^{[\xi]}(x, y), M_{+}^{[\xi]}(x, y)\right]=m_{\left(\xi_{1}, \ldots, \xi_{n}\right)}\left(\operatorname{conv}\left(\operatorname{Fix}\left(m_{\left(\varepsilon_{1}, \ldots, \varepsilon_{p}\right)}\right)\right),\right.
$$

where $\operatorname{conv}(A)$ denotes the convex hull of a set $A$. Thus, naming

$$
E=\operatorname{conv}\left(\operatorname{Fix}\left(m_{\left(\varepsilon_{1}, \ldots, \varepsilon_{p}\right)}\right)\right.
$$

and $\varepsilon=\left(\varepsilon_{1}, \ldots, \varepsilon_{p}\right)$, we have

$$
\sum\left|m_{\left(\xi_{1}, \ldots, \xi_{n}\right)}(E)\right| \leq|y-x|,
$$

where the sum of the left hand-side is taken over all $\left(\xi_{1}, \ldots, \xi_{n}\right) \in \mathcal{F} \mathcal{S}\{0,1\} \backslash$ $\{\varepsilon, \varepsilon \& \varepsilon, \varepsilon \& \varepsilon \& \varepsilon, \ldots\}$ and $|[a, b]|=b-a$ denotes the length of the interval $[a, b]$.

Before continuing the study of the continuity of the map $\delta \mapsto M_{-}^{[\delta]}(x, y)$, a mean with discontinuous iterations is exhibited in the following example.

Example 14 (A mean defined on $I=[0,1]$ with $\delta \mapsto M_{-}^{[\delta]}(0,1)$ discontinuous at rational points) Let define two maps $\mu_{0}, \mu_{1}:[0,1] \rightarrow[0,1]$ by

$$
\mu_{0}(u)=\left\{\begin{array}{cll}
\frac{1}{5} u & \text { if } \quad 0 \leq u \leq \frac{5}{8} \\
u-\frac{1}{2} & \text { if } \quad \frac{5}{8} \leq u \leq 1
\end{array}\right.
$$

and

$$
\mu_{1}(u)=\left\{\begin{array}{ccc}
u+\frac{1}{2} & \text { if } \quad 0 \leq u \leq \frac{3}{8} \\
\frac{1}{5} u+\frac{4}{5} & \text { if } \quad \frac{3}{8} \leq u \leq 1
\end{array} .\right.
$$

Prop. 30 from the Appendix shows that there exist a strictly isotone, symmetric and continuous mean $M$ on $[0,1]$ such that, for every $u \in[0,1]$,

$$
m_{0}(u)=M(0, u)=\mu_{0}(u) \text { and } m_{1}(u)=M(u, 1)=\mu_{1}(u) .
$$

The composition $m_{0} \circ m_{1}$ is promptly computed:

$$
\left(m_{0} \circ m_{1}\right)(u)=\left\{\begin{array}{cll}
\frac{1}{5} u+\frac{1}{10} & \text { if } & 0 \leq u \leq \frac{1}{8} \\
u & \text { if } & \frac{1}{8} \leq u \leq \frac{3}{8} \\
\frac{1}{5} u+\frac{3}{10} & \text { if } & \frac{3}{8} \leq u \leq 1
\end{array}\right.
$$

hence, $\operatorname{Fix}\left(m_{0} \circ m_{1}\right)=[1 / 8,3 / 8]$ and therefore, Theor. 13 shows that every rational point $\xi$ with binary expansion of the form $\left(0 . \xi_{1} \cdots \xi_{n} \overline{01}\right)_{2}$ is a discontinuity point of $\delta \mapsto M_{-}^{[\delta]}(0,1)$. 
A separately quasicontractive mean $M$ give rise to a family of maps $\left\{m_{(\varepsilon)}\right.$ : $\left.\varepsilon=\left(\varepsilon_{1}, \ldots, \varepsilon_{p}\right) \in \mathcal{N} \mathcal{R} \mathcal{F} \mathcal{S}\{0,1\}\right\}$ with a unique fixed point in $I_{(x, y)}$. In fact, if $M$ is separately quasicontractive then, for every $u, u^{\prime} \in I_{(x, y)}$, one at least of the inequalities

$$
\begin{aligned}
& \left|m_{0}(u)-m_{0}\left(u^{\prime}\right)\right|=\left|M(x, u)-M\left(x, u^{\prime}\right)\right|<\left|u-u^{\prime}\right|, \\
& \left|m_{1}(u)-m_{1}\left(u^{\prime}\right)\right|=\left|M(u, y)-M\left(u^{\prime}, y\right)\right|<\left|u-u^{\prime}\right|,
\end{aligned}
$$

hold, and therefore, for $\varepsilon=\left(\varepsilon_{1}, \ldots, \varepsilon_{p}\right) \in \mathcal{N} \mathcal{R} \mathcal{F} \mathcal{S}\{0,1\}$,

$$
\begin{aligned}
& \left|m_{\varepsilon_{1}} \circ \cdots \circ m_{\varepsilon_{n}}(u)-m_{\varepsilon_{1}} \circ \cdots \circ m_{\varepsilon_{n}}\left(u^{\prime}\right)\right| \\
\leq & \left|m_{\varepsilon_{2}} \circ \cdots \circ m_{\varepsilon_{n}}(u)-m_{\varepsilon_{2}} \circ \cdots \circ m_{\varepsilon_{n}}\left(u^{\prime}\right)\right| \\
\leq & \cdots \leq\left|u-u^{\prime}\right|,
\end{aligned}
$$

being strict at least one of these inequalities, so that the existence of two fixed points $u_{1} \neq u_{2}$ has a contradictory consequence:

$$
\left|u_{2}-u_{1}\right|=\left|m_{(\varepsilon)}\left(u_{2}\right)-m_{(\varepsilon)}\left(u_{1}\right)\right|<\left|u_{2}-u_{1}\right| .
$$

Theorem $15 \delta \mapsto M_{-}^{[\delta]}(x, y)$ turns out to be continuous on $[0,1] \cap \mathbb{Q}$ provided that $M$ is a strictly monotonic and separately $(C)$-quasicontractive mean.

Proof. If $M$ is separately (C)-quasicontractive, then $M_{f}$ is separately quasicontractive for a certain homeomorphism $f: I \rightarrow \mathbb{R}$; thus, the previous discussion and Theor. 13 show that $\delta \mapsto\left(M_{f}\right)_{-}^{[\delta]}(x, y)$ is continuous on $[0,1] \cap \mathbb{Q}$ for every $x, y \in f(I)$. Now, $\left(M_{f}\right)^{[d]}=\left(M^{[d]}\right)_{f}, d \in \operatorname{Dyad}([0,1])$, by Prop. 3 and therefore $\left(M_{f}\right)_{-}^{[\delta]}=\left(M_{-}^{[\delta]}\right)_{f}, \delta \in[0,1]$, by the continuity of $f$. Thus, $\delta \mapsto M_{-}^{[\delta]}=\left(\left(M_{f}\right)_{-}^{[\delta]}\right)_{f^{-1}}$ is continuous on $[0,1] \cap \mathbb{Q}$ for every $x, y \in I$.

\section{Continuity at irrational points}

As a first fact concerning the continuity of $\delta \mapsto M_{-}^{[\delta]}(x, y)$ at irrational points it should be noted that the set of irrational discontinuities of this map is at most numerable. Now, let $\xi$ be an irrational number belonging to the interval $[0,1]$; then, for every $n \in \mathbb{N}$ there exits $0 \leq k_{n}<n$ such that

$$
\frac{k_{n}}{2^{n}}<\xi<\frac{k_{n}+1}{2^{n}}
$$

Clearly, $k_{n}=\sum_{i=1}^{n} \xi_{i} 2^{n-i}$ provided that $\left(0 . \xi_{1} \xi_{2} \cdots\right)_{2}$ is the binary expansion of $\xi$ and

$$
\frac{k_{n}}{2^{n}} \uparrow \xi \text { and } \frac{k_{n}+1}{2^{n}} \downarrow \xi
$$


when $n \uparrow+\infty$. In this way, substituting $\varepsilon$ by $\left(\xi_{1}, \xi_{2}, \cdots, \xi_{n}\right)$ successively in (31) and (32) yield

$$
m_{\left(\xi_{1}, \xi_{2}, \cdots, \xi_{n}\right)}(x)=M^{\left[\frac{k_{n}}{2^{n}}\right]}(x, y), x, y \in I,
$$

and

$$
m_{\left(\xi_{1}, \xi_{2}, \cdots, \xi_{n}\right)}(y)=M^{\left[\frac{k_{n}+1}{2^{n}}\right]}(x, y), x, y \in I .
$$

Now, if $M$ is strictly isotone, then, when $x<y$,

$$
\lim _{n \uparrow+\infty} M^{\left[\frac{k_{n}}{2^{n}}\right]}(x, y)=M_{-}^{[\xi]}(x, y) \text { and } \lim _{n \uparrow+\infty} M^{\left[\frac{k_{n}+1}{2^{n}}\right]}(x, y)=M_{+}^{[\xi]}(x, y),
$$

while, when $x>y$,

$$
\lim _{n \uparrow+\infty} M^{\left[\frac{k_{n}}{2^{n}}\right]}(x, y)=M_{+}^{[\xi]}(x, y) \text { and } \lim _{n \uparrow+\infty} M^{\left[\frac{k_{n}+1}{2^{n}}\right]}(x, y)=M_{-}^{[\xi]}(x, y),
$$

and the following result can be established:

Theorem 16 Let $M$ be a strictly isotone mean defined on $I$ and $\xi \in[0,1]$ be an irrational number with binary expansion $\left(0 . \xi_{1} \xi_{2} \cdots\right)_{2}$. Then, for a given $(x, y) \in I^{2}$, the map $\delta \mapsto M_{-}^{[\delta]}(x, y)$ is continuous at $\delta=\xi$ if and only if the sequence $\left\{m_{\left(\xi_{1}, \xi_{2}, \cdots, \xi_{n}\right)}: n \in \mathbb{N}\right\}$ converges to a constant map when $n \uparrow+\infty$.

After the observation made at the beginning of the section it turns out to be that, with the possible exception of a sequence of irrational points, the sequence $\left\{m_{\left(\xi_{1}, \xi_{2}, \cdots, \xi_{n}\right)}: n \in \mathbb{N}\right\}$ always converges to a constant map when $n \uparrow+\infty$. Lemma 9 shows that the hypothesis of the above theorem are fulfilled when $h_{\left(\xi_{1}, \xi_{2}, \cdots, \xi_{n}\right)}$ is defined by (21)-(22) (in whose case $M=A$ ).

Proof. By Prop. 10-ii), the map $m_{\left(\xi_{1}, \xi_{2}, \cdots, \xi_{n}\right)}$ is strictly increasing on $I_{(x, y)}$ when $x \neq y$; thus, assuming $x<y$, it turns out to be

$$
m_{\left(\xi_{1}, \xi_{2}, \cdots, \xi_{n}\right)}(x)<m_{\left(\xi_{1}, \xi_{2}, \cdots, \xi_{n}\right)}(u)<m_{\left(\xi_{1}, \xi_{2}, \cdots, \xi_{n}\right)}(y),
$$

for every $u \in I_{(x, y)}$. If $\delta \mapsto M_{-}^{[\delta]}(x, y)$ is continuous at $\delta=\xi$, then $M_{-}^{[\xi]}(x, y)=$ $M_{+}^{[\xi]}(x, y)$ and taking into account (40), (41) and (42), a passage to the limit $n \uparrow+\infty$ in the above inequalities gives

$$
M_{-}^{[\xi]}(x, y) \leq \lim _{n \uparrow+\infty} m_{\left(\xi_{1}, \xi_{2}, \cdots, \xi_{n}\right)}(u) \leq M_{-}^{[\xi]}(x, y),
$$

so that there exists $\lim _{n \uparrow+\infty} m_{\left(\xi_{1}, \xi_{2}, \cdots, \xi_{n}\right)}(u)$ for every $u \in I_{(x, y)}$ and the limit function does not depend on $u$. When $x>y$, all above inequalities hold in reverse sense, so that the conclusion remains unchanged.

Conversely, if the sequence $\left\{m_{\left(\xi_{1}, \xi_{2}, \cdots, \xi_{n}\right)}: n \in \mathbb{N}\right\}$ converge to a constant map when $n \uparrow+\infty$, then $\lim _{n \uparrow+\infty} m_{\left(\xi_{1}, \xi_{2}, \cdots, \xi_{n}\right)}(x)=\lim _{n \uparrow+\infty} m_{\left(\xi_{1}, \xi_{2}, \cdots, \xi_{n}\right)}(y)$ and therefore, (40), (41) and (42) show that $M_{-}^{[\xi]}(x, y)=M_{+}^{[\xi]}(x, y)$. 
In practice, the convergence to a constant of the sequence $\left\{m_{\left(\xi_{1}, \xi_{2}, \cdots, \xi_{n}\right)}: n \in \mathbb{N}\right\}$ is barely verifiable. In order to prove an operative sufficient condition of continuity of $\delta \mapsto M_{-}^{[\delta]}(x, y)$ at irrational points let us introduce some suitable notation. Recall that the (one-sided) shift transformation $S:[0,1] \rightarrow[0,1]$ is defined by (cf., for instance, [16], pg. 48)

$$
S(t)=2 t(\bmod 1)= \begin{cases}2 t, & 0 \leq t<1 / 2 \\ 2 t-1, & 1 / 2 \leq t \leq 1\end{cases}
$$

and that, if $\left(0 . \xi_{1} \xi_{2} \cdots\right)_{2}$ is the binary expansion of $\xi \in[0,1]$, then $S(\xi)=$ $\left(0 . \xi_{2} \xi_{3} \cdots\right)_{2}$. Now, for a strictly isotone and separately continuous mean $M$, define a map $\phi: I_{(x, y)} \rightarrow I_{(x, y)}$ by

$$
\phi(u)= \begin{cases}m_{0}^{-1}(u), & u \in[x, M(x, y)) \\ m_{1}^{-1}(u), & u \in[M(x, y), y]\end{cases}
$$

when $x<y$, and

$$
\phi(u)= \begin{cases}m_{1}^{-1}(u), & u \in[y, M(x, y)] \\ m_{0}^{-1}(u), & u \in(M(x, y), x]\end{cases}
$$

when $x>y$. Since the maps $m_{0}$ and $m_{1}$ are both strictly increasing and continuous, the definition of $\phi$ is justified by the inequality (28). When $x<$ $y, \phi$ turns out to be strictly increasing and continuous on each subinterval $[x, M(x, y))$ and $[M(x, y), y]$ and, moreover, $\phi(u)>u, u \in(x, M(x, y))$, and $\phi(u)<u, u \in(M(x, y), y]$. Similar properties are enjoyed by $\phi$ when $x>y$.

Finally, for every $\xi \in[0,1]$, denote by $G[\xi]$ the gap of the map $\delta \mapsto M_{-}^{[\delta]}(x, y)$ at the point $\delta=\xi$; i.e.

$$
G[\xi]=\left[M_{-}^{[\xi]}(x, y), M_{+}^{[\xi]}(x, y)\right] .
$$

Lemma 17 Let $M$ be a strictly isotone mean; then, for every $\delta \in[0,1]$,

$$
\phi(G[\delta])=G[S(\delta)] .
$$

In words, the gap at the point $S(\delta)$ is the image under the map $\phi$ of the gap at the point $\delta$.

Proof. In the course of the proof of Theor. 8-ii) was proved that, for every $\delta \in(0,1]$,

$$
M_{-}^{\left[\frac{\delta}{2}\right]}(x, y)=M\left(x, M_{-}^{[\delta]}(x, y)\right) \text { and } M_{-}^{\left[\frac{\delta+1}{2}\right]}(x, y)=M\left(M_{-}^{[\delta]}(x, y), y\right) .
$$

Analogously, if $\delta \in[0,1)$ and $\left(d_{n}^{\prime}: n \in \mathbb{N}\right) \subseteq \operatorname{Dyad}([0,1])$ such that $d_{n}^{\prime} \downarrow \delta$ when $n \uparrow+\infty$, then, (18) holds for $d_{n}^{\prime}$ instead of $d_{n}$, so that a passage to the limit $n \uparrow+\infty$ yields

$$
M_{+}^{\left[\frac{\delta}{2}\right]}(x, y)=M\left(x, M_{+}^{[\delta]}(x, y)\right) \text { and } M_{+}^{\left[\frac{\delta+1}{2}\right]}(x, y)=M\left(M_{+}^{[\delta]}(x, y), y\right) .
$$


The above equalities can be equivalently written as follows:

$$
M_{-}^{[\delta]}(x, y)= \begin{cases}m_{0}\left(M_{-}^{[2 \delta]}(x, y)\right), & \text { if } \delta \in[0,1 / 2) \\ m_{1}\left(M_{-}^{[2 \delta-1]}(x, y)\right), & \text { if } \delta \in[1 / 2,1]\end{cases}
$$

and

$$
M_{+}^{[\delta]}(x, y)=\left\{\begin{array}{ll}
m_{0}\left(M_{+}^{[2 \delta]}(x, y)\right), & \text { if } \delta \in[0,1 / 2) \\
m_{1}\left(M_{+}^{[2 \delta-1]}(x, y)\right), & \text { if } \delta \in[1 / 2,1]
\end{array} .\right.
$$

In this way,

$$
G(2 \delta)=m_{0}^{-1}(G[\delta])
$$

when $\delta \in[0,1 / 2)$, while

$$
G(2 \delta-1)=m_{1}^{-1}(G[\delta])
$$

when $\delta \in[1 / 2,1]$.

Now, observing that

$$
G[\delta] \subseteq \begin{cases}{[x, M(x, y)),} & \text { if } \delta \in[0,1 / 2) \\ {[M(x, y), y],} & \text { if } \delta \in[1 / 2,1]\end{cases}
$$

when $x<y$, and that

$$
G[\delta] \subseteq \begin{cases}(M(x, y), x], & \text { if } \delta \in[0,1 / 2) \\ {[y, M(x, y)],} & \text { if } \delta \in[1 / 2,1]\end{cases}
$$

when $x>y$, it turns out to be that (51) and (52) can be compactly written in the form (48).

Theorem 18 Let $M$ be a strictly isotone and separately $(C)$-nonexpansive mean defined on $I$; then, the maps $\delta \mapsto M_{-}^{[\delta]}(x, y), x, y \in I$, are continuous at every irrational point of $[0,1]$.

Proof. First suppose that $M$ is strictly isotone and separately nonexpansive and fix a point $(x, y) \in I^{2}$. Then, for every $u, u^{\prime} \in I$,

$$
\left|m_{0}(u)-m_{0}\left(u^{\prime}\right)\right| \leq\left|u-u^{\prime}\right| \text { and }\left|m_{1}(u)-m_{1}\left(u^{\prime}\right)\right| \leq\left|u-u^{\prime}\right|,
$$

a pair of inequalities which (taking into account that $m_{0}$ and $m_{1}$ are both strictly increasing functions and, moreover, that

$$
m_{0}([\min \{x, y\}, \max \{x, y\}])=[\min \{x, M(x, y)\}, \max \{x, M(x, y)\}]
$$

and

$$
m_{1}([\min \{x, y\}, \max \{x, y\}])=[\min \{M(x, y), y\}, \max \{M(x, y), y\}]
$$

for every $x, y \in I$ ) can be equivalently written as

$$
\left|m_{0}^{-1}(u)-m_{0}^{-1}\left(u^{\prime}\right)\right| \geq\left|u-u^{\prime}\right|, u, u^{\prime} \in[\min \{x, M(x, y)\}, \max \{x, M(x, y)\}],
$$


and

$$
\left|m_{1}^{-1}(u)-m_{1}^{-1}\left(u^{\prime}\right)\right| \geq\left|u-u^{\prime}\right|, u, u^{\prime} \in[\min \{M(x, y), y\}, \max \{M(x, y), y\}] .
$$

Using these inequalities it can be easily shown that, for every $\delta \in[0,1]$,

$$
|\phi(G[\delta])| \geq|G[\delta]|,
$$

whence, using Lemma 17, the following inequality is derived:

$$
|G[S(\delta)]| \geq|G[\delta]|, \delta \in[0,1] .
$$

Now, suppose that $\xi \in[0,1]$ is an irrational number with binary expansion given by $\left(0 . \xi_{1} \xi_{2} \cdots\right)_{2}$. If the equality $S^{m}(\xi)=S^{n}(\xi)$ was true for $m, n \in \mathbb{N}, m<n$, then, setting $p=n-m$, the equality $S^{k p}\left(S^{n}(\xi)\right)=S^{n}(\xi)$ would be true for every $k \in \mathbb{N}$ and therefore, $S^{n}(\xi)=\left(0 . \xi_{n+1} \xi_{n+2} \cdots\right)_{2}$ would have the form $\left(0 . \overline{\varepsilon_{1} \cdots \varepsilon_{p}}\right)_{2}$. In other terms, $\left(0 . \xi_{1} \cdots \xi_{n} \overline{\varepsilon_{1} \cdots \varepsilon_{p}}\right)_{2}$ would be the form of the binary expansion of $\xi$, contradicting its supposed irrationality. In consequence, $S^{m}(\xi) \neq S^{n}(\xi)$ when $n \neq m$ and therefore, the positive orbit $\left\{S^{n}(\xi): n \in \mathbb{N}_{0}\right\}$ of an irrational number $\xi$ contains an infinity of different points. In this way, if an irrational $\xi \in[0,1]$ was a discontinuity of the map $\delta \mapsto M_{-}^{[\delta]}(x, y)$, then $|G[\xi]|>0$ and, iterating inequality (53), it is deduced

$$
\left|G\left[S^{n}(\delta)\right]\right| \geq|G[\delta]|>0, n \in \mathbb{N},
$$

which, taking into account that $\sum_{n=1}^{+\infty}\left|G\left[S^{n}(\delta)\right]\right| \leq|y-x|<+\infty$, turns out to be a contradiction. Thus, $\delta \mapsto M_{-}^{[\delta]}(x, y)$ can not be discontinuous at any irrational point, as asserted.

When $M$ is separately (C)-nonexpansive then, for a certain homeomorphism $f$, the map $\delta \mapsto\left(M_{f}\right)_{-}^{[\delta]}(x, y)=\left(M_{-}^{[\delta]}\right)_{f}(x, y)$ turns out to be continuous at every irrational point, so that the same is true for the map $\delta \mapsto M_{-}^{[\delta]}(x, y)$. The proof follows from the arbitrariness of the point $(x, y) \in I^{2}$.

Remark 19 (Sufficient condition of continuity for $\delta \mapsto M_{-}^{[\delta]}(x, y)$ at irrational points) A version of the last theorem can be established which provides, when $(x, y) \in I^{2}$ is fixed, a sufficient condition of continuity of the map $\delta \mapsto M_{-}^{[\delta]}(x, y)$ at irrational points. Indeed, if $M$ is strictly isotone and the inequalities

$$
\left|M(x, u)-M\left(x, u^{\prime}\right)\right| \leq\left|u-u^{\prime}\right| \text { and }\left|M(u, y)-M\left(u^{\prime}, y\right)\right| \leq\left|u-u^{\prime}\right|
$$

are satisfied for every $u, u^{\prime} \in I_{(x, y)}$, then $\delta \mapsto M_{-}^{[\delta]}(x, y)$ is continuous at every irrational point of $[0,1]$. For instance, the functions $\mu_{0}$ and $\mu_{1}$ in Example 1 are Lipschitz-continuous with Lipschitz constant equal to 1 , so that inequalities (54) hold for the corresponding mean $M$ at the point $(0,1)$ and therefore, $\delta \mapsto$ $M_{-}^{[\delta]}(0,1)$ is, in this case, continuous at every irrational point. An analogous remark can be made when the inequalities (54) are satisfied by a certain $f$ conjugated $M_{f}$ of $M$. 


\section{Another approach to continuity}

Let $M$ be a strictly isotone and separately continuous mean defined on $I$. Taking into account the definition (45)-(46) of the map $\phi$, the equality (49) can be rewritten in the form

$$
\phi \circ M_{-}^{[\delta]}(x, y)=M_{-}^{[S(\delta)]}(x, y) .
$$

This equality enable us to consider the question of continuity of the map $\delta \mapsto$ $M_{-}^{[\delta]}(x, y)$ from a dynamical point of view.

Firstly, let us write the equality (55) in the form

$$
\phi \circ h_{(x, y)}=h_{(x, y)} \circ S,
$$

where $h_{(x, y)}(\delta)=M_{-}^{[\delta]}(x, y), \delta \in[0,1]$. It is clear from (56) that if $\delta \mapsto$ $M_{-}^{[\delta]}(x, y)=h_{(x, y)}(\delta)$ was continuous, then $h_{(x, y)}$ would be an homeomorphism from $[0,1]$ onto $I_{(x, y)}$ and therefore, $\phi$ would turn out to be topologically conjugated ([16], pg. 68) to the shift $S$. Furthermore, $h_{(x, y)}$ is an increasing homeomorphism when $x<y$ while it is decreasing when $x>y$. Since the converse is also true, the following result can be established:

Theorem 20 Let $M$ be a strictly isotone and separately continuous mean defined on $I$. Then, the map $\delta \mapsto M_{-}^{[\delta]}(x, y)$ is continuous on $[0,1]$ if and only if, for every $x, y \in Y, x \neq y$, the map $\phi$ given by (45)-(46) is topologically conjugated to the shift transformation $S$ through an homeomorphism $H_{(x, y)}$ such that $H_{(x, y)}$ is increasing when $x<y$ and decreasing when $x>y$.

Proof. The necessity was proved in the discussion preceding the statement of the theorem. Conversely, suppose that for every $x, y \in I, x \neq y$, there exists a homeomorphism $h_{(x, y)}$ from $[0,1]$ onto $I_{(x, y)}$ with $h_{(x, y)}$ increasing when $x<y$ and decreasing when $x>y$, such that equality (56) is satisfied by $h_{(x, y)}$; i.e.,

$$
\phi\left(h_{(x, y)}(\delta)\right)=\left\{\begin{array}{cc}
h_{(x, y)}(2 \delta), & 0 \leq \delta<1 / 2 \\
h_{(x, y)}(2 \delta-1), & 1 / 2 \leq \delta \leq 1
\end{array} .\right.
$$

In this way, assuming that $x<y$, it turns out to be $\phi\left(h_{(x, y)}(\delta)\right)>h_{(x, y)}(\delta)$ when $0<\delta<1 / 2$, while $\phi\left(h_{(x, y)}(\delta)\right)<h_{(x, y)}(\delta)$ when $1 / 2 \leq \delta<1$ and thus, from (45) it is deduced that

$$
h_{(x, y)}(\delta) \in\left\{\begin{array}{ll}
{[x, M(x, y)),} & 0 \leq \delta<1 / 2 \\
(M(x, y), y], & 1 / 2 \leq \delta \leq 1
\end{array},\right.
$$

whence

$$
\phi\left(h_{(x, y)}(\delta)\right)=\left\{\begin{array}{ll}
m_{0}^{-1}\left(h_{(x, y)}(\delta)\right), & 0 \leq \delta<1 / 2 \\
m_{1}^{-1}\left(h_{(x, y)}(\delta)\right), & 1 / 2 \leq \delta<1
\end{array} .\right.
$$

From (57), (58) and (26) it is deduced

$$
h_{(x, y)}(\delta)=\left\{\begin{array}{ll}
M\left(x, h_{(x, y)}(2 \delta)\right), & 0 \leq \delta<1 / 2 \\
M\left(h_{(x, y)}(2 \delta-1), y\right), & 1 / 2 \leq \delta \leq 1
\end{array},\right.
$$


which, taking into account that $h_{(x, y)}(0)=x$ and $h_{(x, y)}(1)=y$, shows that $h_{(x, y)}(d)=M^{[d]}(x, y)$ for every $d \in \operatorname{Dyad}([0,1])$. Hence $M_{-}^{[\delta]}(x, y)=h_{(x, y)}(\delta)=$ $M_{-}^{[\delta]}(x, y)$ for every $\delta \in[0,1]$ and $\delta \mapsto M_{-}^{[\delta]}(x, y)$ turns out to be a continuous map. A similar reasoning produces the same equality (59) when $x>y$, so that the proof is completed.

Before finishing this section, a series of remarks and examples is given.

Remark 21 (Theorem 20 for maps on the circle $S^{1}$ ). An equivalent statement of Theor. 20 can be given when the maps entering in equality (56) are considered as being maps on the real numbers $\bmod 1$; i.e., on $\mathbb{R} \backslash \mathbb{Z} \approx S^{1}$. With this purpose, for every $(x, y) \in I^{2}$ define the map $\sigma_{(x, y)}:[0,1] \rightarrow[\min \{x, y\}, \max \{x, y\}]$ by

$$
\sigma_{(x, y)}(\delta)=(1-\delta) \min \{x, y\}+\delta \max \{x, y\}, \delta \in[0,1],
$$

and set $\Phi_{(x, y)}=\sigma_{(x, y)}^{-1} \circ \phi \circ \sigma_{(x, y)}$ and $H_{(x, y)}=\sigma_{(x, y)}^{-1} \circ h_{(x, y)}$. Using these notations, equality (55) takes the form

$$
\Phi_{(x, y)} \circ H_{(x, y)}=H_{(x, y)} \circ S,
$$

in which $\Phi_{(x, y)}, H_{(x, y)}$ and $S$ are maps from $[0,1]$ into itself, so that by identifying the extreme points of the interval $[0,1]$, all maps entering in (55) become maps defined on the circle $S^{1}$. As a map from $S^{1}$ into $S^{1}$, the shift $S$ turns out to be continuous and the same is true for $\Phi_{(x, y)}, x, y \in I$ (since $M$ was supposed strictly isotone and separately continuous). Furthermore, both maps have degree ([16], pg. 72) equal to 2 ; i.e. $\operatorname{deg}\left(\Phi_{(x, y)}\right)=2=\operatorname{deg}(S)$, while

$$
\operatorname{deg}\left(H_{(x, y)}\right)=\left\{\begin{array}{ll}
1, & x<y \\
-1, & x>y
\end{array} .\right.
$$

Theor. 20 can be restated in the following form: for a strictly isotone and separately continuous mean $M$ defined on $I$, the map $\delta \mapsto M_{-}^{[\delta]}(x, y)$ is continuous on $[0,1]$ if and only if, for every $x, y \in Y, x \neq y$, the above defined map $\Phi_{(x, y)}$ is topologically conjugated to the shift $S$ through an homeomorphism $H_{(x, y)}$ whose degree satisfies (62). Moreover, the statement is simplified when $M$ is a symmetric mean: $\delta \mapsto M_{-}^{[\delta]}(x, y)$ is continuous on $[0,1]$ if and only if, for every $x, y \in Y, x<y$, the above defined map $\Phi_{(x, y)}$ is topologically conjugated to the shift $S$ through an homeomorphism $H_{(x, y)}$ with $\operatorname{deg}\left(H_{(x, y)}\right)=1$. In fact, if $x>y$, then interchanging the roles of $x$ and $y$ in (61) yields

$$
\left(\Phi_{(y, x)} \circ H_{(y, x)}\right)(\delta)=\left(H_{(y, x)} \circ S\right)(\delta), \delta \in S^{1},
$$

and thus

$$
\left(\Phi_{(y, x)} \circ H_{(y, x)}\right)(1-\delta)=\left(H_{(y, x)} \circ S\right)(1-\delta), \delta \in S^{1} .
$$

Taking into account that $S(1-\delta)=1-S(\delta), \delta \in S^{1}$ and that, by (a passage to the limit in) Prop. ${ }^{2}$-iv), $M_{-}^{[\delta]}(y, x)=M_{+}^{[1-\delta]}(x, y)$, it is deduced that 
$H_{(x, y)}^{*}(\delta)=H_{(y, x)}(1-\delta)=\sigma_{(y, x)}^{-1}\left(M_{-}^{[1-\delta]}(y, x)\right)=\sigma_{(y, x)}^{-1}\left(M_{+}^{[\delta]}(x, y)\right)$ is a continuous map with degree $\operatorname{deg}\left(H_{(x, y)}^{*}\right)=-1$. Now, in view of $\sigma_{(y, x)}=\sigma_{(x, y)}$ and (45)-(46), $\Phi_{(y, x)}=\sigma_{(y, x)}^{-1} \circ \phi \circ \sigma_{(y, x)}=\sigma_{(x, y)}^{-1} \circ \phi \circ \sigma_{(x, y)}=\Phi_{(x, y)}$ and therefore,

$$
\left(\Phi_{(x, y)} \circ H_{(x, y)}^{*}\right)(\delta)=\left(H_{(x, y)}^{*} \circ S\right)(\delta), \delta \in S^{1} .
$$

Remark 22 (Difference among Aczél's and Ryll-Nardzewski's dyadic iterations) By using the representation given by (15), an equality can be written which is to the Aczél's dyadic iterations as equality (55) is to the Ryll-Nardzewski's ones. Indeed, defining a transformation $T_{(\delta)}: I^{2} \rightarrow I^{2}$ by

$$
T_{(\delta)}(x, y)=\left\{\begin{array}{ll}
T_{0}(x, y), & \delta \in[0,1 / 2) \\
T_{1}(x, y), & \delta \in[1 / 2,1]
\end{array}, x, y \in I,\right.
$$

where $T_{0}, T_{1}: I^{2} \rightarrow I^{2}$ are the mean-type maps respectively given by

$$
T_{0}(x, y)=(x, M(x, y)) \text { and } T_{1}(x, y)=(M(x, y), y), x, y \in I \text {, }
$$

it turns out to be

$$
M_{-}^{(\delta)}=M_{-}^{(S(\delta))} \circ T_{(\delta)} .
$$

The qualitative differences evidenced by (15) and (63) explains the different behaviors of these iterations.

Example 23 (A mean $M$ defined on $I=[0,1]$ with $\delta \mapsto M_{-}^{[\delta]}(0,1)$ discontinuous at an irrational point $\xi \in[0,1])$ If $\xi \in[0,1]$ is an irrational number, then the orbit $O(\xi)$ of $\xi$ under the shift transformation; i.e., $O(\xi)=$ $\left\{S^{n}(\{\xi\}): n \in \mathbb{Z}\right\}$, has the following form: $O(\xi)=A \cup \cup_{n \in \mathbb{N}} B_{-n}$, where $A=O_{+}(\xi)=\left\{\alpha_{n}: \alpha_{n}=S^{n}(\{\xi\}), n \in \mathbb{N}_{0}\right\}$ is the positive orbit of $\xi$ and the negative orbit $O_{-}(\xi)=\bigcup_{n \in \mathbb{N}} B_{-n}$ splits in the (mutually disjoint) sets $B_{-n}=\left\{x \in[0,1]: S^{n}(x)=\xi\right\}$. After (21), (22) and (44), the set of solutions to the equation $S^{n}(x)=\xi$ can be written in the form $\left\{\beta_{n, k}: 0 \leq k<2^{n}\right\}$ where $\beta_{n, k}$ is given by

$$
\beta_{n, k}=h_{(\varepsilon)}(\xi),
$$

provided that $\varepsilon=\left(\varepsilon_{1}, \ldots, \varepsilon_{n}\right) \in \mathcal{F S}\{0,1\}$ satisfies $(\ell(\varepsilon)=n$ and $) \sum_{i=1}^{n} \varepsilon_{i} 2^{n-i}=$ $k$. Now, for a doubly infinite sequence $\left(\gamma_{n}: n \in \mathbb{Z}\right) \subseteq \mathbb{R}^{+}$such that $\sum_{n=-\infty}^{+\infty} \gamma_{n}=$ 1 let us consider the function $H:[0,1] \rightarrow[0,1]$ defined by $H(0)=0$ and

$$
H(u)=\frac{1}{2}\left(u+\sum_{\alpha_{n} \leq u} \gamma_{n}+\sum_{\beta_{n, k} \leq u} \frac{\gamma_{n}}{2^{n}}\right), u \in(0,1]
$$

Clearly, $H$ is a strictly increasing and left continuous function whose unique discontinuities are the points of the orbit $O(\xi)$. The set $J=[0,1] \backslash \overline{H([0,1])}$ is an open subset of $\mathbb{R}$ with an infinite number of components; more precisely, $J=\bigcup_{n \in \mathbb{N}_{0}} J_{n} \cup \bigcup_{n \in \mathbb{N}, 0 \leq k<2^{n}} J_{-n}^{(k)}$ where $J_{n}=\left(H\left(\alpha_{n}^{-}\right), H\left(\alpha_{n}^{+}\right)\right), n \in \mathbb{N}_{0}$, and 
$J_{-n}^{(k)}=\left(H\left(\beta_{n, k}^{-}\right), H\left(\beta_{n, k}^{+}\right)\right), n \in \mathbb{N}, 0 \leq k<2^{n}$. Now, define a map $f:[0,1] \rightarrow$ $[0,1]$ by

$$
f(u)=\left\{\begin{array}{ll}
H(S(v)), \text { if } u=H(v) \text { with } v \in[0,1] \backslash O(\xi) & \\
H\left((S(v))^{-}\right)+\frac{\left(u-H\left(v^{-}\right)\right)\left(H\left((S(v))^{+}\right)-H\left((S(v))^{-}\right)\right)}{H\left(v^{+}\right)-H\left(v^{-}\right)}, & \text {if } u \in\left[H\left(v^{-}\right), H\left(v^{+}\right)\right]
\end{array},\right.
$$

and realize that $f$ really has the form

$$
f(u)=\left\{\begin{array}{ll}
f_{0}(u), & u \in[0, H(1 / 2)) \\
f_{1}(u), & u \in[H(1 / 2), 1]
\end{array},\right.
$$

where the maps $f_{0}$ and $f_{1}$ are strictly increasing and continuous on $[0, H(1 / 2))$ and $[H(1 / 2), 1]$, respectively. Additionally, $f_{0}(u)>u, 0<u<H(1 / 2)$, and $f_{1}(u)<u, H(1 / 2)<u<1$, while $f_{0}\left((H(1 / 2))^{-}\right)=1$ and $f_{1}(H(1 / 2))=0$ and therefore, the hypotheses of Prop. 30 are satisfied by the pair of maps $\mu_{0}=f_{0}^{-1}, \mu_{1}=f_{1}^{-1}$. In conclusion, there exists a strictly isotone, symmetric and continuous mean $M$ on $[0,1]$ such that, for every $u \in[0,1]$,

$$
M(0, u)=\mu_{0}(u) \text { and } M(u, 1)=\mu_{1}(u) .
$$

Since the equality $\phi \circ H=H \circ S$ holds for this mean and $H$ is strictly increasing and left continuous, it turns out to be $H(\delta)=M_{-}^{[\delta]}(0,1), \delta \in[0,1]$, so that $\delta \mapsto M_{-}^{[\delta]}(0,1)$ is discontinuous at the point $\xi$.

\section{Weightings by using Ryll-Nardzewski's dyadic iterations}

The results proved in the preceding sections enable us to establish the following theorems on weightings based on Ryll-Nardzewski's dyadic iterations.

Theorem 24 Let $M$ be a strictly isotone and separately continuos mean defined on I. In order that the map $\delta \mapsto M_{-}^{[\delta]}(x, y)$ be continuous for a given $(x, y) \in I$ it is necessary and sufficient that the following conditions be satisfied:

(i) for every $\varepsilon=\left(\varepsilon_{1}, \ldots, \varepsilon_{n}\right) \in \mathcal{N} \mathcal{R} \mathcal{F} \mathcal{S}\{0,1\}$, the map $m_{(\varepsilon)}=m_{\varepsilon_{1}} \circ \cdots \circ m_{\varepsilon_{n}}$ has a unique fixed point in $I_{(x, y)}$; and

(ii) for every irrational number $\xi=\left(0 . \xi_{1} \xi_{2} \cdots\right)_{2} \in[0,1]$, the sequence of maps $\left\{m_{\left(\xi_{1}, \xi_{2}, \cdots, \xi_{n}\right)}: n \in \mathbb{N}\right\}$ converges to a constant in $I_{(x, y)}$ when $n \uparrow+\infty$.

When conditions (i) and (ii) hold for every $(x, y) \in I^{2}$, the maps $\delta \mapsto$ $M_{-}^{[\delta]}(x, y), x, y \in I$, are all strictly monotonic and continuous on $[0,1]$. Moreover, $M_{-}^{[0]}(x, y)=0, M_{-}^{[1 / 2]}(x, y)=M(x, y)$ and $M_{-}^{[1]}(x, y)=y$, so that $\delta \mapsto M_{-}^{[\delta]}$ is a continuous and strictly monotonic weighting of the mean $M$. The mean $M_{-}^{[\delta]}$ turns out to be continuous for every $\delta \in[0,1]$ provided that $M$ is continuous. 
Proof. After Theors. 13 and 16, only the continuity of $M_{-}^{[\delta]}$ on $I^{2}$ requires some discussion. When $M$ is a continuous mean, $M^{[d]}$ is continuous for every $d \in \operatorname{Dyad}([0,1])$ and taking into account $(17), M_{-}^{[\delta]}$ turns out to be lower semicontinuous when $x<y$ and upper semicontinuous when $x>y$. Analogously, $M_{+}^{[\delta]}$ turns out to be upper semicontinuous when $x<y$ and lower semicontinuous when $x>y$. Now, $M_{-}^{[\delta]}=M_{+}^{[\delta]}$ by the continuity of $\delta \mapsto M_{-}^{[\delta]}(x, y)$ and therefore, $M_{-}^{[\delta]}$ is continuous.

Theorem 25 Let $M$ be a strictly isotone and separately continuos mean defined on I. In order that the map $\delta \mapsto M_{-}^{[\delta]}(x, y)$ be continuous for a given $(x, y) \in I$ it is necessary and sufficient that the map $\phi$ given by (45)-(46) be topologically conjugated to the shift transformation $S$ through an homeomorphism $H_{(x, y)}$ such that $H_{(x, y)}$ is increasing when $x<y$ and decreasing when $x>y$. When this condition is fulfilled by $\phi$, the map $\delta \mapsto M_{-}^{[\delta]}$ turns out to be a continuous and strictly monotonic weighting of the mean $M$. The mean $M_{-}^{[\delta]}$ turns out to be continuous for every $\delta \in[0,1]$ provided that $M$ is continuous.

Proof. The proof follows immediately from Theor. 20. As for the continuity of $M_{-}^{[\delta]}$, it follows from the same argument employed in the proof of Theor. 24 .

Theorem 26 Let $M$ be a strictly isotone and separately (C)-quasicontractive mean defined on $I$; then the maps $\delta \mapsto M_{-}^{[\delta]}(x, y), x, y \in I$, are strictly monotonic and continuous on $[0,1]$. Moreover, $\delta \mapsto M_{-}^{[\delta]}$ is a continuous and strictly monotonic weighting of the mean $M$. The mean $M_{-}^{[\delta]}$ turns out to be continuous for every $\delta \in[0,1]$.

Proof. The proof of the assertions of the theorem is an immediate consequence of Theors. 15, 18. Since a separately (C)-quasicontractive mean is a Lipschitzcontinuous function, the continuity of $M_{-}^{[\delta]}$ is proved as in the proof of the previous theorem.

It should be added that, when $M$ is a symmetric mean satisfying the hypotheses of any one of the preceding theorems, then the weighting $\delta \mapsto M_{-}^{[\delta]}$ of $M$ possesses the following symmetry property:

$$
M_{-}^{[1-\delta]}(x, y)=M_{-}^{[1-\delta]}(y, x), x, y \in I, \delta \in[0,1] .
$$

\section{Appendix}

A useful criterion of separated nonexpansiveness or quasicontractiveness can be formulated in terms of partial derivatives. To this end, consider a mean $M$ defined on $I$ such that there exist its partial derivatives $M_{x}$ and $M_{y}$ on $I^{2}$. If $M$ is, in addition, separately nonexpansive, then passing to the limit $u^{\prime} \rightarrow u$ in the inequalities (12) yields

$$
\left|M_{x}\right| \leq 1 \text { and }\left|M_{y}\right| \leq 1 .
$$


Conversely, if a mean $M$ has partial derivatives $M_{x}$ and $M_{y}$ satisfying inequalities (64), then the Mean Value Theorem yields

$$
\left|M(x, u)-M\left(x, u^{\prime}\right)\right|=\left|M_{y}(x, \theta)\right|\left|u-u^{\prime}\right| \leq\left|u-u^{\prime}\right|, u, u^{\prime} \in I,
$$

where $\theta$ is an intermediate value, and in a similar way,

$$
\left|M(u, y)-M\left(u^{\prime}, y\right)\right| \leq\left|u-u^{\prime}\right|, u, u^{\prime} \in I,
$$

so that $M$ turns out to be separately nonexpansive. Clearly, $M$ turns out to be separately quasicontractive provided that at least one of the inequalities (64) is strict.

Proposition 27 Let $M$ be a mean defined on I with partial derivatives existing at every point $(x, y) \in I^{2}$; then $M$ is separately nonexpansive if and only if the inequalities (64) are satisfied by $M_{x}$ and $M_{y}$. When one at least of these inequalities is strict, $M$ turns out to be separately quasicontractive.

Proof. See the previous discussion.

Using the above result, an example is exhibited which shows that separate (C)-nonexpansiveness is a property weaker than separate nonexpansiveness.

Example 28 (A separately (C)-quasicontractive but not separately nonexpansive mean). The Heronian mean $\mathfrak{H}_{\mathfrak{E}}$ is the mean defined (cf. [13], pg. 399) by

$$
\mathfrak{H}_{\mathfrak{E}}(x, y)=\frac{x+y+\sqrt{x y}}{3}, x, y>0 .
$$

Since $\left(\mathfrak{H}_{\mathfrak{E}}\right)_{x}(x, y)=(1+\sqrt{y / x} / 2) / 3>0$ and $\left(\mathfrak{H}_{\mathfrak{E}}\right)_{y}(x, y)=(1+\sqrt{x / y} / 2) / 3>$ $0, \mathfrak{H}_{\mathfrak{E}}$ is strictly isotone. Moreover, $\left\{(x, y) \in \mathbb{R}^{+} \times \mathbb{R}^{+}:\left(\mathfrak{H}_{\mathfrak{E}}\right)_{x}(x, y) \leq 1\right.$ and $\left.\left(\mathfrak{H}_{\mathfrak{E}}\right)_{y}(x, y) \leq 1\right\}=\left\{(x, y) \in \mathbb{R}^{+} \times \mathbb{R}^{+}: 1 / 16 \leq y / x \leq 16\right\} \neq \mathbb{R}^{+} \times \mathbb{R}^{+}$, and therefore $\mathfrak{H}_{\mathfrak{E}}$ is not a separately nonexpansive mean by Prop. 27. The conjugated $\left(\mathfrak{H}_{\mathfrak{E}}\right)_{f}$ of $\mathfrak{H}_{\mathfrak{E}}$ by the homeomorphism $f(x)=\sqrt{x}, x>0$, is clearly given by

$$
\left(\mathfrak{H}_{\mathfrak{E}}\right)_{f}(x, y)=\sqrt{\frac{x^{2}+x y+y^{2}}{3}}, x, y>0 .
$$

The mean $\left(\mathfrak{H}_{\mathfrak{E}}\right)_{f}=\mathcal{L}^{[2]}$ is known as the generalized logarithmic mean of order 2 (cf. [13], pg. 385) and, as expected, $\mathcal{L}_{x}^{[2]}(x, y), \mathcal{L}_{y}^{[2]}(x, y)>0, x, y>0$, so that $\mathcal{L}^{[2]}$ is also strictly isotone. Now, by adding the partial derivatives of $\mathcal{L}^{[2]}$, for $x, y>0$ it is obtained

$$
\begin{aligned}
\mathcal{L}_{x}^{[2]}(x, y), \mathcal{L}_{y}^{[2]}(x, y) & <\mathcal{L}_{x}^{[2]}(x, y)+\mathcal{L}_{y}^{[2]}(x, y) \\
& =\left(\sqrt{\frac{x^{2}+x y+y^{2}}{3}}\right)^{-1}\left(\frac{x+y}{2}\right)=\frac{A(x, y)}{\mathcal{L}^{[2]}(x, y)} \leq 1 .
\end{aligned}
$$

The last inequality is derived from the fact that $\mathcal{L}^{[2]}$ is a superarithmetic mean:

$$
\mathcal{L}^{[2]}(x, y) \geq A(x, y), x, y>0 .
$$

In this way, Prop. 27 shows that $\mathcal{L}^{[2]}$ is separately quasicontractive. 
Even if separate $(\mathrm{C})$-nonexpansiveness is a property considerably weaker than separate nonexpansiveness, it is far from being generally enjoyed by continuous means. The next example illustrates this fact.

Example 29 (A family of continuous but not separately (C)-nonexpansive means) Suppose that a continuous mean $M$ defined on I satisfies the following property: there exists an isolated point $\left(x_{0}, y_{0}\right) \in \operatorname{int}\left(I^{2}\right), x_{0} \neq y_{0}$, such that $M\left(x_{0}, y_{0}\right)=\min \left\{x_{0}, y_{0}\right\}$ or $M\left(x_{0}, y_{0}\right)=\max \left\{x_{0}, y_{0}\right\}$; in other words, $M$ ceases of being a strict mean only in an isolated point $\left(x_{0}, y_{0}\right) \in \operatorname{int}\left(I^{2}\right)$. Let us show that $M$ can not be separately $(C)$-nonexpansive. In fact, if $M$ was separately (C)-nonexpansive, then there would exist a homeomorphism $f: I \rightarrow \mathbb{R}$ such that the inequalities

$$
\left|f(M(x, u))-f\left(M\left(x, u^{\prime}\right)\right)\right| \leq\left|f(u)-f\left(u^{\prime}\right)\right|
$$

and

$$
\left|f(M(u, y))-f\left(M\left(u^{\prime}, y\right)\right)\right| \leq\left|f(u)-f\left(u^{\prime}\right)\right|
$$

hold for every $x, y, u, u^{\prime} \in I$. Suppose that $x_{0}<y_{0}, M\left(x_{0}, y_{0}\right)=x_{0}$ and that, for a small enough $\delta>0,\left(x_{0}, y_{0}\right)$ is the unique (not diagonal!) point in the square $\left[x_{0}-\delta, y_{0}+\delta\right]^{2}$ with this property. In this way, the two points $u_{1}=x_{0}$ and $u_{2}=y_{0}$ are the unique fixed points of the function $m_{0}:\left[x_{0}, y_{0}+\delta\right] \rightarrow$ $\left[x_{0}, y_{0}+\delta\right]$ defined by $m_{0}(u)=M\left(x_{0}, u\right)$. Furthermore, $m_{0}(u)<u$ for every $u \in\left(x_{0}, y_{0}\right) \cup\left(y_{0}, y_{0}+\delta\right)$ and therefore, the sequences $\left\{m_{0}^{n}\left(y_{0}-\delta_{0}\right): n \in \mathbb{N}\right\}$ and $\left\{m_{0}^{n}\left(y_{0}+\delta_{0}\right): n \in \mathbb{N}\right\}$ with $0<\delta_{0}<\min \left\{\delta, y_{0}-x_{0}\right\}$ turns out to be monotonic and

$$
m_{0}^{n}\left(y_{0}-\delta_{0}\right) \downarrow x_{0}, \quad m_{0}^{n}\left(y_{0}+\delta_{0}\right) \downarrow y_{0}
$$

when $n \uparrow+\infty$. Now, setting $x=x_{0}$ in the inequality (65) produces

$$
\left.\mid f\left(m_{0}(u)\right)-f\left(m_{0}\left(u^{\prime}\right)\right)\right)|\leq| f(u)-f\left(u^{\prime}\right) \mid, u, u^{\prime} \in\left[x_{0}, y_{0}+\delta\right],
$$

whence the inequality

$$
\left|f\left(m_{0}^{n}(u)\right)-f\left(m_{0}^{n}\left(u^{\prime}\right)\right)\right| \leq\left|f(u)-f\left(u^{\prime}\right)\right|, u, u^{\prime} \in\left[x_{0}, y_{0}+\delta\right]
$$

follows by iteration. Replacing in this last equality $u$ by $y_{0}-\delta_{0} / 2$, $u^{\prime}$ by $y_{0}+\delta_{0} / 2$ and then passing to the limit $n \uparrow+\infty$, it is deduced

$$
\begin{aligned}
\left|f\left(x_{0}\right)-f\left(y_{0}\right)\right| & =\lim _{n \uparrow+\infty}\left|f\left(m_{0}^{n}\left(y_{0}-\delta_{0}\right)\right)-f\left(m_{0}^{n}\left(y_{0}+\delta_{0}\right)\right)\right| \\
& \leq\left|f\left(y_{0}-\delta_{0}\right)-f\left(y_{0}+\delta_{0}\right)\right|, 0<\delta_{0}<\min \left\{\delta, y_{0}-x_{0}\right\} .
\end{aligned}
$$

Since $f$ is injective, $\left|f\left(x_{0}\right)-f\left(y_{0}\right)\right|>0$ and then, the continuity of $f$ at $y_{0}$ is contradicted by the above inequality. In the case in which $x_{0}<y_{0}, M\left(x_{0}, y_{0}\right)=$ $y_{0}$, a similar contradiction is obtained by considering the map $m_{1}(u)=M\left(u, y_{0}\right)$ in the interval $\left[x_{0}-\delta, y_{0}\right]$ and inequality (66). The remaining cases are analogously treated. This proves that $M$ can not be separately $(C)$-nonexpansive. 
Since the mean $M$ constructed in Example 23 is discontinuous at the points of the orbit of an irrational number, it can not be separately (C)-nonexpansive by Theor. 18; thus, an example of strict mean which is not separately (C)nonexpansive is provided by $M$.

Now, let us prove the result used in constructing the means of Examples 14 and 23 .

Proposition 30 Let $\mu_{0}, \mu_{1}:[0,1] \rightarrow[0,1]$ be two continuous and strictly increasing functions such that $\mu_{0}(u)<u<\mu_{1}(u)$ for all $u \in(0,1)$, and $\mu_{0}(0)=0, \mu_{1}(1)=1, \mu_{0}(1)=\alpha=\mu_{1}(0)$ with $0<\alpha<1$; then, there exists a strictly isotone, symmetric and continuous mean $M$ on $[0,1]$ such that, for every $u \in[0,1]$,

$$
M(0, u)=\mu_{0}(u) \text { and } M(u, 1)=\mu_{1}(u) .
$$

Of course, there are infinite continuous symmetric means $M$ defined on $[0,1]$ satisfying the boundary value conditions (67). For instance, if $\mu$ is the harmonic function in the triangle $0<u<v<1$ with boundary values given by $\mu(0, t)=$ $\mu_{0}(t), \mu(t, 1)=\mu_{1}(t), \mu(t, t)=t,(0 \leq t \leq 1)$, then, by the maximum principle (cf. [15]), $u \leq \mu(u, v) \leq v$ for every $u<v$, whence the symmetric extension to $[0,1]^{2}$ of $\mu$, which is given by

$$
M(u, v)=\left\{\begin{array}{ll}
\mu(u, v), & u \leq v \\
\mu(v, u), & v \leq u
\end{array},\right.
$$

turns out to be a symmetric continuous mean defined on $[0,1]$. The interest of the above proposition lies in the existence of a strictly isotone and continuous mean satisfying the boundary conditions of the statement.

Proof. In order to prove the proposition it is sufficient to define a strictly isotone and continuous mean $M$ on the triangular domain $T=\left\{(u, v) \in[0,1]^{2}: u \leq v\right\}$. Indeed, extending $M$ to the whole square $[0,1]^{2}$ by $M(u, v)=M(v, u)$ gives a strictly isotone, symmetric and continuous mean $M$ defined on $[0,1]$.

Let us consider a splitting of the triangle $T$ in four triangular subdomains $T_{i}, i=1,2,3,4$, respectively defined by

$$
\begin{aligned}
& T_{1}=\{(u, v) \in T: v \leq \alpha\}, \quad T_{2}=\{(u, v) \in T: v \geq \alpha,(1-\alpha) u+\alpha v \leq \alpha\} \\
& T_{3}=\{(u, v) \in T: u \leq \alpha,(1-\alpha) u+\alpha v \geq \alpha\}, \quad T_{4}=\{(u, v) \in T: u \geq \alpha\}
\end{aligned}
$$

and define $M$ on $T$ as follows:

$$
M(u, v)=\left\{\begin{array}{cc}
\left(1-\frac{u}{v}\right) \mu_{0}(v)+u, & (u, v) \in T_{1} \\
\left(1-\frac{(1-\alpha) u}{\alpha(1-v)}\right) \mu_{0}(v)+\frac{(1-\alpha) u}{1-v}, & (u, v) \in T_{2} \\
\left(1-\frac{\alpha(1-v)}{(1-\alpha) u}\right) \mu_{1}(u)+\frac{\alpha^{2}(1-v)}{(1-\alpha) u}, & (u, v) \in T_{3} \\
\left(\frac{v-u}{1-u}\right) \mu_{1}(u)+\frac{1-v}{1-u} u, & (u, v) \in T_{4}
\end{array} .\right.
$$

For every $i=1,2,3,4$, the function $M_{i}=\left.M\right|_{T_{i}}, i=1,2,3,4$, is continuous and strictly increasing in both variables on its respective domain of definition. 
Take, for example, $i=2$; then, the continuity of $M_{2}$ follows from that of $\mu_{0}$ and, regarding the monotonicity, we have the equality

$$
\begin{aligned}
M_{2}(u, v) & =\left(1-\frac{(1-\alpha) u}{\alpha(1-v)}\right) \mu_{0}(v)+\frac{(1-\alpha) u}{1-v} \\
& =\frac{1-\alpha}{1-v}\left(1-\frac{\mu_{0}(v)}{\alpha}\right) u+\mu_{0}(v),
\end{aligned}
$$

in which, taking into account that $\mu_{0}(v)<\alpha<1$ provided that $v<1$, the inequality

$$
\frac{1-\alpha}{1-v}\left(1-\frac{\mu_{0}(v)}{\alpha}\right)>0
$$

holds for the coefficient of the variable $u$ and thus, $u \mapsto M_{2}(u, v)$ turns out to be strictly increasing in the interval $\left[0, \frac{\alpha(1-v)}{1-\alpha}\right]$. Note that the interval $\left[0, \frac{\alpha(1-v)}{1-\alpha}\right]$ reduces to the point $u=0$ when $v=1$. Now, if $0 \leq u<\alpha$ and $v_{1}, v_{2} \in$ $\left[\alpha, 1-\frac{(1-\alpha) u}{\alpha}\right], v_{1}<v_{2}$, in view of $v \mapsto(1-\alpha) u /(1-v)$ is strictly increasing on $[0,1)$ as well as $\mu_{0}$, we have

$$
\begin{aligned}
M_{2}\left(u, v_{1}\right) & =\left(1-\frac{(1-\alpha) u}{\alpha\left(1-v_{1}\right)}\right) \mu_{0}\left(v_{1}\right)+\frac{(1-\alpha) u}{1-v_{1}} \\
& <\left(1-\frac{(1-\alpha) u}{\alpha\left(1-v_{2}\right)}\right) \mu_{0}\left(v_{1}\right)+\frac{(1-\alpha) u}{1-v_{2}} \\
& <\left(1-\frac{(1-\alpha) u}{\alpha\left(1-v_{2}\right)}\right) \mu_{0}\left(v_{2}\right)+\frac{(1-\alpha) u}{1-v_{2}}=M_{2}\left(u, v_{2}\right),
\end{aligned}
$$

which, taking into account that the interval $\left[\alpha, 1-\frac{(1-\alpha) u}{\alpha}\right]$ reduces to the point $\alpha$ when $u=\alpha$, proves that $u \mapsto M_{2}(u, v)$ is strictly increasing in the interval $\left[\alpha, 1-\frac{(1-\alpha) u}{\alpha}\right]$. The proof of the continuity and the strict monotonicity of the remaining $M_{i}$ is similar.

Now, for every $i=1,2,3,4$, the function $M_{i}$ is internal in its respective domain of definition. For example, taking into account that $0 \leq \mu_{0}(v) \leq \alpha<1$, it can be written

$$
\begin{aligned}
M_{2}(u, v) & \leq\left(1-\frac{(1-\alpha) u}{\alpha(1-v)}\right) \alpha+\frac{(1-\alpha) u}{1-v} \\
& =\alpha \leq v
\end{aligned}
$$

and

$$
M_{2}(u, v) \geq \frac{(1-\alpha) u}{1-v} \geq u
$$

for every $(u, v) \in T_{2}$. The internality of the remaining $M_{i}$ can be analogously proved. Note in passing that it is right here where the hypotheses $\mu_{0}(u)<$ $u<\mu_{1}(u), 0<u<1$, are employed: the inequality $\mu_{0}(v)<v$ shows that $M_{1}(u, v)<v$, while the inequality $u<M_{4}(u, v)$ is a consequence of $u<\mu_{1}(u)$. 
Now, observing that

$$
\begin{aligned}
& T_{1} \cap T_{2}=\{(u, v) \in T: v=\alpha, u \leq \alpha\}, \\
& T_{2} \cap T_{3}=\{(u, v) \in T:(1-\alpha) u+\alpha v=\alpha\}, \\
& T_{3} \cap T_{4}=\{(u, v) \in T: u=\alpha\},
\end{aligned}
$$

while

$$
T_{1} \cap T_{3}=T_{1} \cap T_{4}=T_{2} \cap T_{4}=\{(\alpha, \alpha)\},
$$

it is easy to see that $\left.M_{i}\right|_{T_{i} \cap T_{j}}=\left.M_{j}\right|_{T_{i} \cap T_{j}}$ for every $i, j=1,2,3,4, i \neq j$, and hence, that $M$ defined by (68) turns out to be a strictly isotone and continuous mean $M$ on the triangular domain $T$. This finishes the proof.

Acknowledgment: E. L. Grin read a preliminary version of the paper and contributed with valuable observations, among which Example 23.

\section{References}

[1] J. Aczél, On mean values, Bull. Amer. Math. Soc. 54, (1948), 392-400.

[2] J. Aczél, Lectures on Functional Equations and their Applications, Academic Press, New York and London, (1966).

[3] J. Aczél, J. Dhombres, Functional Equations in Several Variables, Cambridge Univ. Press, Cambridge, 1989.

[4] L. R. Berrone, A dynamical characterization of quasilinear means, Aequationes Math. 84, Issue 1 (2012), 51-70.

[5] L. R. Berrone, The Aumann functional equation for general weighting procedures, Aequationes Math., DOI 10.1007/s00010-015-0344-4, (2015).

[6] L. R. Berrone, Generalized Cauchy means, Aequationes Math., DOI 10.1007/s00010-015-0341-7, (2015).

[7] L. R. Berrone, Weighting general means, (to appear).

[8] L. R. Berrone, A. L. Lombardi, A note on equivalence of means, Publ. Math. Debrecen 58, Fasc. 1-2, (2001), 49-56.

[9] L. R. Berrone, J. Moro, Lagrangian means, Aequationes Math. 55, (1998), $217-226$.

[10] L. R. Berrone, J. Moro, On means generated through the Cauchy mean value theorem, Aequationes Math. 60, (2000), 1-14.

[11] L. R. Berrone, G. E. Sbérgamo, La familia de bases de una media continua y la representación de las medias cuasiaritméticas, Bol. de la Asoc. Venezolana de Matemática, Vol. XIX, No. 1, (2012), 3-18. 
[12] L. R. Berrone, G. E. Sbérgamo, Weightings general means by iteration, (to appear).

[13] P. S. Bullen, Handbook of Means and Their Inequalities, Kluwer Academic Publishers, Dordrecht, 2010.

[14] P. S. Bullen, D. S. Mitrinović, P. M. Vasić, Means and Their Inequalities, D. Reidel Publishing Company, Dordrecht, 1988.

[15] D. Gilbarg, N. S. Trudinger, Elliptic Partial Differential Equations of Second Order, Springer, Berlin, (1983).

[16] A. Katok, B. Hasselblatt, Introduction to the Modern Theory of Dynamical Systems, Encyclopedia of Math. and its Appl. v. 54, Cambridge Univ. Press, Cambridge, 1995.

[17] A. Katok, B. Hasselblatt, A First Course in Dynamics (with a Panorama of Recent Developments), Cambridge Univ. Press, Cambridge, 2003.

[18] J. Matkowski, On weighted extensions of Bajraktarević means, Sarajevo J. Math. 6, No. 19, (2010), 169-188.

[19] M. Raïssouli, Parameterized logarithmic mean, Int. Journal of Math. Analysis, Vol. 6 no. 18, (2012), 863-869.

[20] M. Raïssouli, J. Sándor, On a method of construction of new means with applications, Journal of Inequalities and Applications, DOI 10.1186/1029242X-2013-89, (2013).

[21] K. C. Richards, H. C. Tiedeman, A note on weighted identric and logarithmic means, J. Ineq. Pure and Appl. Math. 7(5) Art. 157, (2006).

[22] C. Ryll-Nardzewski, Sur les moyennes, Studia Math. 11, (1949), 31-37. 